Colloquium Mathematicum 139(2015), no.2, 273-298

\title{
Turán's problem and Ramsey numbers for trees
}

\author{
Zhi-Hong Sun ${ }^{1}$, Lin-Lin Wang ${ }^{2}$ and Yi-Li Wu ${ }^{3}$ \\ ${ }^{1}$ School of Mathematical Sciences, Huaiyin Normal University, \\ Huaian, Jiangsu 223001, P.R. China \\ Email: zhihongsun@yahoo.com \\ Homepage: http://www.hytc.edu.cn/xsjl/szh \\ ${ }^{2}$ School of Science, China University of Mining and Technology, \\ Xuzhou, Jiangsu 221116, PR China \\ Email: wanglinlin-1986@aliyun.com \\ ${ }^{3}$ School of Mathematical Sciences, Huaiyin Normal University, \\ Huaian, Jiangsu 223001, PR China \\ Email: yiliwu@126.com
}

\begin{abstract}
Let $T_{n}^{1}=\left(V, E_{1}\right)$ and $T_{n}^{2}=\left(V, E_{2}\right)$ be the trees on $n$ vertices with $V=\left\{v_{0}, v_{1}, \ldots, v_{n-1}\right\}$, $E_{1}=\left\{v_{0} v_{1}, \ldots, v_{0} v_{n-3}, v_{n-4} v_{n-2}, v_{n-3} v_{n-1}\right\}$, and $E_{2}=\left\{v_{0} v_{1}, \ldots, v_{0} v_{n-3}, v_{n-3} v_{n-2}, v_{n-3}\right.$ $\left.v_{n-1}\right\}$. In this paper, for $p \geq n \geq 5$ we obtain explicit formulas for $\operatorname{ex}\left(p ; T_{n}^{1}\right)$ and $\operatorname{ex}\left(p ; T_{n}^{2}\right)$, where $\operatorname{ex}(p ; L)$ denotes the maximal number of edges in a graph of order $p$ not containing $L$ as a subgraph. Let $r\left(G_{1}, G_{2}\right)$ be the Ramsey number of the two graphs $G_{1}$ and $G_{2}$. In this paper we also obtain some explicit formulas for $r\left(T_{m}, T_{n}^{i}\right)$, where $i \in\{1,2\}$ and $T_{m}$ is a tree on $m$ vertices with $\Delta\left(T_{m}\right) \leq m-3$.
\end{abstract}

2010 Mathematics Subject Classification: 05C55, 05C35, 05C05

Key words and phrases: Ramsey number, tree, Turán's problem

\section{Introduction}

In this paper, all graphs are simple graphs. For a graph $G=(V(G), E(G))$ let $e(G)=$ $|E(G)|$ be the number of edges in $G$ and let $\Delta(G)$ be the maximal degree of $G$. For a forbidden graph $L$, let $\operatorname{ex}(p ; L)$ denote the maximal number of edges in a graph of order $p$ not containing any copies of $L$. The corresponding Turán problem is to evaluate $\operatorname{ex}(p ; L)$. For a graph $G$ of order $p$, if $G$ does not contain any copies of $L$ and $e(G)=\operatorname{ex}(p ; L)$, we say that $G$ is an extremal graph. In this paper we also use $\operatorname{Ex}(p ; L)$ to denote the set of extremal graphs of order $p$ not containing $L$ as a subgraph.

Let $\mathbb{N}$ be the set of positive integers. Let $p, n \in \mathbb{N}$ with $p \geq n \geq 2$. For a given tree $T_{n}$ on $n$ vertices, it is difficult to determine the value of $\operatorname{ex}\left(p ; T_{n}\right)$. The famous Erdös-Sós conjecture asserts that $\operatorname{ex}\left(p ; T_{n}\right) \leq \frac{(n-2) p}{2}$. For the progress on the Erdös-Sós conjecture, see for example $[8,11]$. Write $p=k(n-1)+r$, where $k \in \mathbb{N}$ and $r \in\{0,1, \ldots, n-2\}$. Let $P_{n}$ be the path on $n$ vertices. In [4] Faudree and Schelp showed that

$$
\operatorname{ex}\left(p ; P_{n}\right)=k\left(\begin{array}{c}
n-1 \\
2
\end{array}\right)+\left(\begin{array}{l}
r \\
2
\end{array}\right)=\frac{(n-2) p-r(n-1-r)}{2} .
$$


Let $K_{1, n-1}$ denote the unique tree on $n$ vertices with $\Delta\left(K_{1, n-1}\right)=n-1$, and let $T_{n}^{\prime}$ denote the unique tree on $n$ vertices with $\Delta\left(T_{n}^{\prime}\right)=n-2$. For $n \geq 4$ let $T_{n}^{*}=(V, E)$ be the tree on $n$ vertices with $V=\left\{v_{0}, v_{1}, \ldots, v_{n-1}\right\}$ and $E=\left\{v_{0} v_{1}, \ldots, v_{0} v_{n-3}, v_{n-3} v_{n-2}, v_{n-2} v_{n-1}\right\}$. In [10] we determine $\operatorname{ex}\left(p ; K_{1, n-1}\right), \operatorname{ex}\left(p ; T_{n}^{\prime}\right)$ and $\operatorname{ex}\left(p ; T_{n}^{*}\right)$. For $i=1,2$ let $T_{n}^{i}=\left(V, E_{i}\right)$ be the tree on $n$ vertices with

$$
\begin{aligned}
& V=\left\{v_{0}, v_{1}, \ldots, v_{n-1}\right\}, \\
& E_{1}=\left\{v_{0} v_{1}, \ldots, v_{0} v_{n-3}, v_{n-4} v_{n-2}, v_{n-3} v_{n-1}\right\}, \\
& E_{2}=\left\{v_{0} v_{1}, \ldots, v_{0} v_{n-3}, v_{n-3} v_{n-2}, v_{n-3} v_{n-1}\right\} .
\end{aligned}
$$

In this paper, for $p \geq n \geq 5$ we obtain explicit formulas for $\operatorname{ex}\left(p ; T_{n}^{1}\right)$ and $\operatorname{ex}\left(p ; T_{n}^{2}\right)$ (see Theorems 2.1 and 3.1).

For a graph $G$, as usual $\bar{G}$ denotes the complement of $G$. Let $G_{1}$ and $G_{2}$ be two graphs. The Ramsey number $r\left(G_{1}, G_{2}\right)$ is the smallest positive integer $p$ such that, for every graph $G$ with $p$ vertices, either $G$ contains a copy of $G_{1}$ or else $\bar{G}$ contains a copy of $G_{2}$.

Let $n \in \mathbb{N}, n \geq 6$ and let $T_{n}$ be a tree on $n$ vertices. As mentioned in [7], recently Zhao proved the following conjecture of Burr and Erdős [2]: $r\left(T_{n}, T_{n}\right) \leq 2 n-2$. Let $m, n \in \mathbb{N}$. In 1973 Burr and Roberts [3] showed that for $m, n \geq 3$,

$$
r\left(K_{1, m-1}, K_{1, n-1}\right)= \begin{cases}m+n-3 & \text { if } 2 \nmid m n, \\ m+n-2 & \text { if } 2 \mid m n .\end{cases}
$$

In 1995, Guo and Volkmann [5] proved that for $n>m \geq 4$,

$$
r\left(K_{1, m-1}, T_{n}^{\prime}\right)= \begin{cases}m+n-3 & \text { if } 2 \mid m(n-1), \\ m+n-4 & \text { if } 2 \nmid m(n-1) .\end{cases}
$$

Recently the first author evaluated the Ramsey number $r\left(T_{m}, T_{n}^{*}\right)$ for $T_{m} \in\left\{P_{m}, K_{1, m-1}\right.$, $\left.T_{m}^{\prime}, T_{m}^{*}\right\}$. In particular, he proved that (see [9]) for $n>m \geq 7$,

$$
r\left(K_{1, m-1}, T_{n}^{*}\right)= \begin{cases}m+n-3 & \text { if } m-1 \mid n-3, \\ m+n-4 & \text { if } m-1 \nmid n-3 .\end{cases}
$$

Suppose $m, n \in \mathbb{N}$ and $i, j \in\{1,2\}$. In this paper, using the formula for $\operatorname{ex}\left(p ; T_{n}^{i}\right)$ and the method in [9] we evaluate $r\left(T_{m}, T_{n}^{i}\right)$ for $T_{m} \in\left\{K_{1, m-1}, T_{m}^{\prime}, T_{m}^{*}, T_{m}^{j}\right\}$. In particular, we have the following typical results:

$$
\begin{aligned}
& r\left(T_{n}^{i}, T_{n}^{j}\right)=2 n-6-\left(1-(-1)^{n}\right) / 2, r\left(P_{n}, T_{n}^{j}\right)=2 n-7 \text { for } n \geq 17, \\
& r\left(T_{n}^{i}, T_{n}^{\prime}\right)=r\left(T_{n}^{i}, T_{n}^{*}\right)=2 n-5 \text { for } n \geq 8, \\
& r\left(K_{1, m-1}, T_{n}^{i}\right)=m+n-4 \text { for } n>m \geq 7 \text { and } 2 \mid m n, \\
& r\left(T_{m}^{i}, T_{n}^{j}\right)=m+n-5 \text { for } m \geq 7, n \geq(m-3)^{2}+3 \text { and } m-1 \nmid n-4,
\end{aligned}
$$

and for $n>m \geq 16$,

$$
r\left(T_{m}^{\prime}, T_{n}^{i}\right)= \begin{cases}m+n-4 & \text { if } m-1 \mid n-4 \\ m+n-6 & \text { if } n=m+1 \equiv 1(\bmod 2) \\ m+n-5 & \text { otherwise }\end{cases}
$$


In addition to the notation introduced above, throughout the paper we also use the following symbols: $[x]$ is the greatest integer not exceeding $x, d(v)$ is the degree of the vertex $v$ in a graph, $\Gamma(v)$ is the set of vertices adjacent to the vertex $v, d(u, v)$ is the distance between the two vertices $u$ and $v$ in a graph, $K_{n}$ is the complete graph on $n$ vertices, $G\left[V_{0}\right]$ is the subgraph of $G$ induced by vertices in the set $V_{0}$ (we write $G\left[v_{1}, \ldots, v_{m}\right]$ instead of $\left.G\left[\left\{v_{1}, \ldots, v_{m}\right\}\right]\right), G-V_{0}$ is the subgraph of $G$ obtained by deleting vertices in $V_{0}$ and all edges incident to them, and finally $e\left(V_{1} V_{1}^{\prime}\right)$ is the number of edges with one endpoint in $V_{1}$ and another endpoint in $V_{1}^{\prime}$.

\section{Evaluation of $\operatorname{ex}\left(p ; T_{n}^{1}\right)$}

Lemma 2.1. Let $p, n \in \mathbb{N}$ with $p \geq n-1 \geq 1$. Then $\operatorname{ex}\left(p ; K_{1, n-1}\right)=\left[\frac{(n-2) p}{2}\right]$.

This is a known result. See for example [10, Theorem 2.1].

Lemma 2.2. Let $p, n \in \mathbb{N}, p \geq n \geq 7$ and $G \in E x\left(p ; T_{n}^{1}\right)$. Suppose that $G$ is connected. Then $\Delta(G)=n-4$ and $e(G)=\left[\frac{(n-4) p}{2}\right]$.

Proof. Since a graph not containing $K_{1, n-3}$ as a subgraph implies that the graph does not contain $T_{n}^{1}$ as a subgraph, by Lemma 2.1 we have

$$
e(G)=\operatorname{ex}\left(p ; T_{n}^{1}\right) \geq \operatorname{ex}\left(p ; K_{1, n-3}\right)=\left[\frac{(n-4) p}{2}\right] .
$$

If $\Delta(G) \leq n-5$, using Euler's theorem we see that $e(G)=\frac{1}{2} \sum_{v \in V(G)} d(v) \leq \frac{(n-5) p}{2}$. This together with $(2.1)$ yields $\frac{(n-4) p-1}{2} \leq\left[\frac{(n-4) p}{2}\right] \leq e(G) \leq \frac{(n-5) p}{2}$. This is impossible. Hence $\Delta(G) \geq n-4$. Now we show that $\Delta(G)=n-4$.

Suppose $q \geq n$ and $q=k(n-1)+r$ with $k \in \mathbb{N}$ and $r \in\{0,1, \ldots, n-2\}$. Then clearly $k K_{n-1} \cup K_{r}$ does not contain any copies of $T_{n}^{1}$ and so ex $\left(q ; T_{n}^{1}\right) \geq e\left(k K_{n-1} \cup K_{r}\right)$. For $q=n$ we see that $e\left(k K_{n-1} \cup K_{r}\right)=e\left(K_{n-1} \cup K_{1}\right)=\frac{(n-1)(n-2)}{2}>2 n-1$. For $q \geq n+1$ we see that $(n-6) q \geq(n-6)(n+1)>\left(\frac{n-1}{2}\right)^{2}-2$ and so $e\left(k K_{n-1} \cup K_{r}\right)=\frac{k(n-1)(n-2)}{2}+\frac{r(r-1)}{2}=$ $\frac{(n-2) q-r(n-1-r)}{2} \geq \frac{(n-2) q-\left(\frac{n-1}{2}\right)^{2}}{2}>2 q-1$. Hence

$$
\operatorname{ex}\left(q ; T_{n}^{1}\right) \geq e\left(k K_{n-1} \cup K_{r}\right)>2 q-1 \quad \text { for } q \geq n .
$$

Suppose $v_{0} \in V(G), d\left(v_{0}\right)=\Delta(G)=m$ and $\Gamma\left(v_{0}\right)=\left\{v_{1}, \ldots, v_{m}\right\}$. If $m=p-1$, as $G$ does not contain $T_{n}^{1}$ as a subgraph, we see that $G\left[v_{1}, \ldots, v_{m}\right]$ does not contain $2 K_{2}$ as a subgraph and hence $e\left(G\left[v_{1}, \ldots, v_{m}\right]\right) \leq m-1$. Therefore

$$
e(G)=d\left(v_{0}\right)+e\left(G\left[v_{1}, \ldots, v_{m}\right]\right) \leq m+m-1=2 p-3 .
$$

By (2.2), we have $e(G)=\operatorname{ex}\left(p ; T_{n}^{1}\right)>2 p-1$ and we get a contradiction. Hence $m<$ $p-1$. Suppose that $u_{1}, \ldots, u_{t}$ are all vertices in $G$ such that $d\left(u_{1}, v_{0}\right)=\cdots=d\left(u_{t}, v_{0}\right)=$ 2. Then $t \geq 1$. Assume $u_{1} v_{1} \in E(G)$ with no loss of generality. If $m=p-2$, then $V(G)=\left\{v_{0}, v_{1}, \ldots, v_{m}, u_{1}\right\}$ and $v_{i} v_{j} \notin E(G)$ for $2 \leq i<j \leq m$. If $v_{1} v_{i} \in E(G)$ for some $i \in\{2,3, \ldots, m\}$, then $u_{1} v_{j} \notin E(G)$ for all $j \neq 1, i$. Hence $\operatorname{ex}\left(p ; T_{n}^{1}\right)=e(G) \leq$ $\max \{2 m, m+3\} \leq 2 m=2 p-4$, which contradicts (2.2).

By the above, $m<p-2$. We first assume $m \geq n-2$. As $G$ does not contain any copies of $T_{n}^{1}$, we see that $\left\{v_{2}, \ldots, v_{m}\right\}$ is an independent set, $u_{i} v_{j} \notin E(G)$ for any $i \in\{2,3, \ldots, t\}$ and $j \in\{2,3, \ldots, m\}$, and $u_{i} v_{1} \in E(G)$ for any $i=1,2, \ldots, t$. Set $V_{1}=\left\{v_{0}, v_{2}, v_{3}, \ldots, v_{m}\right\}$. Then 
$e\left(G\left[V_{1}\right]\right)=m-1$. If $u_{1}$ is adjacent to at least two vertices in $\left\{v_{2}, v_{3}, \ldots, v_{m}\right\}$, then $v_{1} v_{j} \notin$ $E(G)$ for any $j=2,3, \ldots, m$. If $v_{1}$ is adjacent to at least two vertices in $\left\{v_{2}, v_{3}, \ldots, v_{m}\right\}$, then $u_{1} v_{j} \notin E(G)$ for any $j=2,3, \ldots, m$. Hence there are at most $m$ edges with one endpoint in $V_{1}$ and another endpoint in $G-V_{1}$. Therefore,

$$
e(G) \leq e\left(G\left[V_{1}\right]\right)+m+e\left(G-V_{1}\right)=2 m-1+e\left(G-V_{1}\right) .
$$

For $m \in\{n-2, n-1\}$ let $G_{1}=K_{m}$. Then clearly $e\left(G_{1}\right)=\frac{m(m-1)}{2}>2 m-1$. For $m=k(n-1)+r \geq n$ with $k \in \mathbb{N}$ and $0 \leq r \leq n-2$ let $G_{1}=k K_{n-1} \cup K_{r}$. Then $G_{1}$ does not contain any copies of $T_{n}^{1}$ and $e\left(G_{1}\right)>2 m-1$ by (2.2). Thus, by (2.4) we have $e(G) \leq 2 m-1+e\left(G-V_{1}\right)<e\left(G_{1} \cup\left(G-V_{1}\right)\right)$ for $m \geq n-2$. This contradicts the fact $G \in \operatorname{Ex}\left(p ; T_{n}^{1}\right)$.

Suppose $m=n-3$ and $d\left(v_{1}\right)=n-3$. Then $v_{1} v_{s} \notin E(G)$ for some $s \in\{2,3, \ldots, n-3\}$. We claim that $V(G)=\left\{v_{0}, v_{1}, \ldots, v_{m}, u_{1}, \ldots, u_{t}\right\}$. Otherwise, there exists $w \in V(G)$ such that $d\left(v_{0}, w\right)=3$. As $d\left(v_{1}\right)=n-3$, we see that the subgraph induced by $\left\{v_{1}, v_{s}, w\right\} \cup \Gamma\left(v_{1}\right)$ contains a copy of $T_{n}^{1}$. This contradicts the assumption $G \in \operatorname{Ex}\left(p ; T_{n}^{1}\right)$. Hence the claim is true and so $|V(G)|=p=n-2+t$. Since $p \geq n$ we have $t \geq 2$. For $i=1,2, \ldots, t$ and $j=2,3, \ldots, n-3$ we have $u_{i} v_{j} \notin E(G), u_{i} v_{1} \in E(G)$ and so $t+1 \leq d\left(v_{1}\right)=n-3$. Therefore $2 \leq t \leq n-4$ and hence

$$
\begin{aligned}
e(G) & =e\left(G\left[v_{0}, v_{2}, v_{3}, \ldots, v_{n-3}\right]\right)+d\left(v_{1}\right)+e\left(G\left[u_{1}, \ldots, u_{t}\right]\right) \\
& \leq\left(\begin{array}{c}
n-3 \\
2
\end{array}\right)+n-3+\left(\begin{array}{c}
t \\
2
\end{array}\right)=\left(\begin{array}{c}
n-2 \\
2
\end{array}\right)+\left(\begin{array}{l}
t \\
2
\end{array}\right) .
\end{aligned}
$$

Clearly $K_{n-1} \cup K_{t-1}$ does not contain $T_{n}^{1}$ and

$$
e\left(K_{n-1} \cup K_{t-1}\right)=\left(\begin{array}{c}
n-1 \\
2
\end{array}\right)+\left(\begin{array}{c}
t-1 \\
2
\end{array}\right)=\left(\begin{array}{c}
n-2 \\
2
\end{array}\right)+\left(\begin{array}{l}
t \\
2
\end{array}\right)+n-1-t>e(G) .
$$

This contradicts the assumption $G \in \operatorname{Ex}\left(n-2+t ; T_{n}^{1}\right)$.

Now suppose $m=n-3$ and $d\left(v_{1}\right) \leq n-4$. If $t=1$, setting $V_{2}=\left\{v_{0}, v_{1}, \ldots, v_{n-3}, u_{1}\right\}$ we see that

$$
\begin{aligned}
e(G) & =e\left(G\left[v_{0}, v_{2}, v_{3}, \ldots, v_{n-3}\right]\right)+d\left(v_{1}\right)+d\left(u_{1}\right)-1+e\left(G-V_{2}\right) \\
& \leq\left(\begin{array}{c}
n-3 \\
2
\end{array}\right)+n-4+n-4+e\left(G-V_{2}\right) \\
& =\frac{n^{2}-3 n-4}{2}+e\left(G-V_{2}\right)<e\left(K_{n-1} \cup\left(G-V_{2}\right)\right) .
\end{aligned}
$$

This contradicts the assumption $G \in \operatorname{Ex}\left(p ; T_{n}^{1}\right)$. Hence $t \geq 2$. For $i=1,2, \ldots, t$ and $j=2,3, \ldots, n-3$ we see that $u_{i} v_{j} \notin E(G)$ and $u_{i} v_{1} \in E(G)$. Let $V_{3}=\left\{v_{0}, v_{1}, \ldots, v_{n-3}\right\}$. Then

$$
\begin{aligned}
e(G) & =d\left(v_{1}\right)+e\left(G\left[v_{0}, v_{2}, v_{3}, \ldots, v_{n-3}\right]\right)+e\left(G-V_{3}\right) \\
& \leq n-4+\left(\begin{array}{c}
n-3 \\
2
\end{array}\right)+e\left(G-V_{3}\right)=\frac{n^{2}-5 n+4}{2}+e\left(G-V_{3}\right) \\
& <e\left(K_{n-2} \cup\left(G-V_{3}\right)\right) .
\end{aligned}
$$

Since $G$ is an extremal graph, we get a contradiction. 
Summarizing all the above we obtain $\Delta(G)=n-4$ and so $e(G)=\sum_{v \in V(G)} d(v) \leq \frac{(n-4) p}{2}$. This together with $(2.1)$ yields $e(G)=\left[\frac{(n-4) p}{2}\right]$, which completes the proof.

Lemma 2.3. Let $n, n_{1}, n_{2} \in \mathbb{N}$ with $n_{1}<n-1$ and $n_{2}<n-1$. Then

$$
\left(\begin{array}{c}
n_{1} \\
2
\end{array}\right)+\left(\begin{array}{c}
n_{2} \\
2
\end{array}\right)<\min \left\{\left(\begin{array}{c}
n_{1}+n_{2} \\
2
\end{array}\right),\left(\begin{array}{c}
n-1 \\
2
\end{array}\right)+\left(\begin{array}{c}
n_{1}+n_{2}-n+1 \\
2
\end{array}\right)\right\} .
$$

Proof. It is clear that

$$
\left(\begin{array}{c}
n_{1} \\
2
\end{array}\right)+\left(\begin{array}{c}
n_{2} \\
2
\end{array}\right)=\frac{\left(n_{1}+n_{2}\right)\left(n_{1}+n_{2}-1\right)-2 n_{1} n_{2}}{2}<\left(\begin{array}{c}
n_{1}+n_{2} \\
2
\end{array}\right)
$$

and

$$
\begin{aligned}
& \left(\begin{array}{c}
n-1 \\
2
\end{array}\right)+\left(\begin{array}{c}
n_{1}+n_{2}-n+1 \\
2
\end{array}\right)-\left(\begin{array}{c}
n_{1} \\
2
\end{array}\right)-\left(\begin{array}{c}
n_{2} \\
2
\end{array}\right) \\
& =\frac{(n-1)(n-2)+\left(n_{1}+n_{2}-n+1\right)\left(n_{1}+n_{2}-n\right)}{2}-\frac{\left(n_{1}+n_{2}\right)\left(n_{1}+n_{2}-1\right)-2 n_{1} n_{2}}{2} \\
& =\left(n-1-n_{1}\right)\left(n-1-n_{2}\right)>0 .
\end{aligned}
$$

Thus the lemma is proved.

Lemma 2.4. Suppose that $p \in \mathbb{N}, p \geq 6$, and $G$ is a connected graph of order $p$ that does not contain any copies of $T_{6}^{1}$. Then $e(G) \leq 2 p-3$.

Proof. Clearly $\Delta\left(T_{6}^{1}\right)=3$. Suppose $v_{0} \in V(G), d\left(v_{0}\right)=\Delta(G)=m$ and $\Gamma\left(v_{0}\right)=$ $\left\{v_{1}, \ldots, v_{m}\right\}$. If $\Delta(G)=m \leq 3$, using Euler's theorem we see that $e(G) \leq \frac{3 p}{2} \leq 2 p-3$. From now on we assume $\Delta(G)=m \geq 4$. If $d(v) \leq 2$ for all $v \in V(G)-\left\{v_{0}\right\}$, then

$$
e(G)=\frac{1}{2} \sum_{v \in V(G)} d(v) \leq \frac{1}{2}(m+2(p-1)) \leq \frac{3(p-1)}{2}<2 p-3 .
$$

So the result is true. Now we assume $d(v) \geq 3$ for some $v \in V(G)-\left\{v_{0}\right\}$. We may choose a vertex $u_{0} \in V(G)$ so that $u_{0} \neq v_{0}, d\left(u_{0}\right) \geq 3$ and $d\left(u_{0}, v_{0}\right)$ is as small as possible.

We first assume $d\left(u_{0}, v_{0}\right)=1$ and $u_{0}=v_{1}$ with no loss of generality. That is, $d\left(v_{1}\right) \geq 3$. Suppose $\Gamma\left(v_{1}\right) \subset\left\{v_{0}, v_{1}, \ldots, v_{m}\right\}$. Since $d\left(v_{1}\right) \geq 3$ and $G$ does not contain any copies of $T_{6}^{1}$, we see that $V(G)=\left\{v_{0}, \ldots, v_{m}\right\}, m=p-1 \geq 5$ and $G\left[v_{1}, \ldots, v_{m}\right]$ does not contain any copies of $2 K_{2}$. Thus $e(G) \leq d\left(v_{0}\right)+m-1=2 m-1 \leq 2(m+1)-3=2 p-3$. Now assume $\Gamma\left(v_{1}\right)-\left\{v_{0}, v_{1}, \ldots, v_{m}\right\}=\left\{w_{1}, \ldots, w_{t}\right\}$. Since $d\left(v_{0}\right)=m \geq 5, d\left(v_{1}\right) \geq 3$ and $G$ does not contain any copies of $T_{6}^{1}$, we see that $V(G)=\left\{v_{0}, v_{1}, \ldots, v_{m}, w_{1}, \ldots, w_{t}\right\}$ and $\left\{v_{2}, \ldots, v_{m}\right\}$ is an independent set. For $t \geq 2$, we have $e\left(G\left[w_{1}, \ldots, w_{t}\right]\right) \leq 1$ and $v_{i} w_{j} \notin E(G)$ for any $i \in\{2,3, \ldots, m\}$ and $j \in\{1,2, \ldots, t\}$. Thus $e(G) \leq d\left(v_{0}\right)+d\left(v_{1}\right)-1+1 \leq 2 m<2(m+1+t)-$ $3=2 p-3$. Now assume $t=1$. Then $v_{1} v_{i} \in E(G)$ for some $i \in\{2,3, \ldots, m\}$ and $v_{j} w_{1} \notin E(G)$ for $j \in\{2,3, \ldots, m\}-\{i\}$. Hence $e(G) \leq d\left(v_{0}\right)+d\left(v_{1}\right)-1+1 \leq 2 m<2(m+2)-3=2 p-3$.

Next we assume $d\left(u_{0}, v_{0}\right)=2$. Then $\left\{v_{1}, \ldots, v_{m}\right\}$ is an independent set. If $\Gamma\left(u_{0}\right) \subseteq$ $\left\{v_{1}, \ldots, v_{m}\right\}$, then $V(G)=\left\{v_{0}, \ldots, v_{m}, u_{0}\right\}$ and so $e(G)=d\left(v_{0}\right)+d\left(u_{0}\right) \leq m+m<2(m+2)-$ $3=2 p-3$. If $\Gamma\left(u_{0}\right)-\left\{v_{2}, \ldots, v_{m}\right\}=\left\{v_{1}, w_{1}, \ldots, w_{t}\right\}$, we see that $V(G)=\left\{v_{0}, v_{1}, \ldots, v_{m}, u_{0}\right.$, $\left.w_{1}, \ldots, w_{t}\right\}$ and so $e(G)=d\left(v_{0}\right)+d\left(u_{0}\right)+e\left(G\left[w_{1}, \ldots, w_{t}\right]\right) \leq m+m+1<2(m+2+t)-3=$ $2 p-3$.

Finally we assume $d\left(u_{0}, v_{0}\right) \geq 3$. Suppose that $v_{0} v_{1} u_{1} u_{2} \cdots u_{k} u_{0}$ is the shortest path in $G$ between $v_{0}$ and $u_{0}$, and $\Gamma\left(u_{0}\right)=\left\{w_{1}, \ldots, w_{t}, u_{k}\right\}$. Since $G$ is connected and $G$ does not contain any copies of $T_{6}^{1}$, it is easily seen that $V(G)=\left\{v_{0}, v_{1}, \ldots, v_{m}, u_{1}, \ldots, u_{k}, u_{0}, w_{1}, \ldots, w_{t}\right\}$, 
$d\left(v_{2}\right)=\cdots=d\left(v_{m}\right)=1, d\left(v_{1}\right)=d\left(u_{1}\right)=\cdots=d\left(u_{k}\right)=2$ and $e\left(G\left[w_{1}, \ldots, w_{t}\right]\right) \leq 1$. Clearly $G$ is a tree or a graph obtained by adding an edge to a tree. Hence $e(G) \leq p<2 p-3$.

Summarizing all the above proves the lemma.

Theorem 2.1. Suppose $p, n \in \mathbb{N}, p \geq n-1 \geq 4$ and $p=k(n-1)+r$, where $k \in \mathbb{N}$ and $r \in\{0,1, \ldots, n-2\}$. Then

$$
\begin{aligned}
\operatorname{ex}\left(p ; T_{n}^{1}\right)= & \max \left\{\left[\frac{(n-2) p}{2}\right]-(n-1+r), \frac{(n-2) p-r(n-1-r)}{2}\right\} \\
= & \begin{cases}{\left[\frac{(n-2) p}{2}\right]-(n-1+r)} & \text { if } n \geq 16 \text { and } 3 \leq r \leq n-6 \text { or if } \\
\frac{(n-2) p-r(n-1-r)}{2} & 13 \leq n \leq 15 \text { and } 4 \leq r \leq n-7, \\
\text { otherwise. }\end{cases}
\end{aligned}
$$

Proof. Clearly ex $\left(n-1 ; T_{n}^{1}\right)=e\left(K_{n-1}\right)=\frac{(n-2)(n-1)}{2}$. Thus the result is true for $p=n-1$. From now on we assume $p \geq n$. Since $T_{5}^{1} \cong P_{5}$, by (1.1) we obtain the result in the case $n=5$. Now we assume $n \geq 6$. Suppose $G \in \operatorname{Ex}\left(p ; T_{n}^{1}\right)$ and $G_{1}, \ldots, G_{t}$ are all components of $G$ with $\left|V\left(G_{i}\right)\right|=p_{i}$ and $p_{1} \leq p_{2} \leq \cdots \leq p_{t}$. Then clearly $G_{i} \in \operatorname{Ex}\left(p_{i} ; T_{n}^{1}\right)$ for $i=1,2, \ldots, t$.

We first consider the case $n=6$. If $p_{i} \leq 5$, then clearly $G_{i} \cong K_{p_{i}}$ and $e\left(G_{i}\right)=\left(\begin{array}{c}p_{i} \\ 2\end{array}\right)$. If $p_{i} \geq 6$ and $p_{i}=5 k_{i}+r_{i}$ with $k_{i} \in \mathbb{N}$ and $0 \leq r_{i} \leq 4$, from Lemma 2.4 we have $e\left(G_{i}\right) \leq 2 p_{i}-3 \leq 2 p_{i}-\frac{r_{i}\left(5-r_{i}\right)}{2}=e\left(k_{i} K_{5} \cup K_{r_{i}}\right)$. Since $k_{i} K_{5} \cup K_{r_{i}}$ does not contain any copies of $T_{6}^{1}$ and $G_{i} \in \operatorname{Ex}\left(p_{i} ; T_{6}^{1}\right)$, we see that $e\left(G_{i}\right) \geq e\left(k_{i} K_{5} \cup K_{r_{i}}\right)$ and so $e\left(G_{i}\right)=e\left(k_{i} K_{5} \cup K_{r_{i}}\right)$. Therefore, there is a graph $G^{\prime} \in \operatorname{Ex}\left(p ; T_{6}^{1}\right)$ such that $G^{\prime}=a_{1} K_{1} \cup a_{2} K_{2} \cup a_{3} K_{3} \cup a_{4} K_{4} \cup a_{5} K_{5}$, where $a_{1}, \ldots, a_{5}$ are nonnegative integers. If $a_{1}+a_{2}+a_{3}+a_{4} \leq 1$, then $\operatorname{ex}\left(p ; T_{6}^{1}\right)=e\left(G^{\prime}\right)=$ $e\left(a_{5} K_{5} \cup K_{r}\right)=k\left(\begin{array}{l}5 \\ 2\end{array}\right)+\left(\begin{array}{l}r \\ 2\end{array}\right)$. If $a_{1}+a_{2}+a_{3}+a_{4}>1$, then $2 a_{1}+3 a_{2}+3 a_{3}+2 a_{4}>3 \geq \frac{r(5-r)}{2}$ and so

$$
\begin{aligned}
& e\left(a_{1} K_{1} \cup a_{2} K_{2} \cup a_{3} K_{3} \cup a_{4} K_{4}\right) \\
& =a_{2}+3 a_{3}+6 a_{4}<2\left(a_{1}+2 a_{2}+3 a_{3}+4 a_{4}\right)-\frac{r(5-r)}{2}=\left(k-a_{5}\right)\left(\begin{array}{l}
5 \\
2
\end{array}\right)+\left(\begin{array}{l}
r \\
2
\end{array}\right) .
\end{aligned}
$$

Thus, $\operatorname{ex}\left(p ; T_{6}^{1}\right)=e\left(G^{\prime}\right)=e\left(a_{1} K_{1} \cup a_{2} K_{2} \cup a_{3} K_{3} \cup a_{4} K_{4}\right)+e\left(a_{5} K_{5}\right)<k\left(\begin{array}{l}5 \\ 2\end{array}\right)+\left(\begin{array}{l}r \\ 2\end{array}\right)$. Since $k K_{5} \cup K_{r}$ does not contain any copies of $T_{6}^{1}$, we get a contradiction. Thus $\operatorname{ex}\left(p ; T_{6}^{1}\right)=$ $e\left(k K_{5} \cup K_{r}\right)=k\left(\begin{array}{l}5 \\ 2\end{array}\right)+\left(\begin{array}{l}r \\ 2\end{array}\right)=2 p-\frac{r(5-r)}{2}$. This proves the result for $n=6$.

From now on we assume $n \geq 7$. If $t=1$, then $G$ is connected. Thus, by Lemma 2.2 we have

$$
e(G)=\left[\frac{(n-4) p}{2}\right] \text { for } t=1 .
$$

Now we assume $t \geq 2$. We claim that $p_{i} \geq n-1$ for $i \geq 2$. Otherwise, $p_{1} \leq p_{2}<n-1$ and so $G_{1} \cup G_{2} \cong K_{p_{1}} \cup K_{p_{2}}$. If $p_{1}+p_{2}<n$, by Lemma 2.3 we have $e\left(G_{1} \cup G_{2}\right)=$ $e\left(K_{p_{1}} \cup K_{p_{2}}\right)=\left(\begin{array}{c}p_{1} \\ 2\end{array}\right)+\left(\begin{array}{c}p_{2} \\ 2\end{array}\right)<\left(\begin{array}{c}p_{1}+p_{2} \\ 2\end{array}\right)=e\left(K_{p_{1}+p_{2}}\right)$. Since $K_{p_{1}+p_{2}}$ does not contain $T_{n}^{1}$ and $G_{1} \cup G_{2} \in \operatorname{Ex}\left(p_{1}+p_{2} ; T_{n}^{1}\right)$ we get a contradiction. Hence $p_{1}+p_{2} \geq n$. Using Lemma 2.3 again we see that

$$
\begin{aligned}
e\left(G_{1} \cup G_{2}\right) & =e\left(K_{p_{1}} \cup K_{p_{2}}\right)=\left(\begin{array}{c}
p_{1} \\
2
\end{array}\right)+\left(\begin{array}{c}
p_{2} \\
2
\end{array}\right) \\
& <\left(\begin{array}{c}
n-1 \\
2
\end{array}\right)+\left(\begin{array}{c}
p_{1}+p_{2}-n+1 \\
2
\end{array}\right)=e\left(K_{n-1} \cup K_{p_{1}+p_{2}-n+1}\right) .
\end{aligned}
$$


Since $p_{1} \leq p_{2}<n-1$, we have $p_{1}+p_{2}-n+1<n-1$. Therefore $K_{n-1} \cup K_{p_{1}+p_{2}-n+1}$ does not contain $T_{n}^{1}$. As $G_{1} \cup G_{2}$ is an extremal graph without $T_{n}^{1}$, we also get a contradiction. Thus, the claim is true.

Next we claim that $p_{i} \leq n-1$ for all $i=1,2, \ldots, t-1$. If $p_{t-1} \geq n$, by Lemma 2.2 we have

$$
e\left(G_{t-1} \cup G_{t}\right)=e\left(G_{t-1}\right)+e\left(G_{t}\right)=\left[\frac{(n-4) p_{t-1}}{2}\right]+\left[\frac{(n-4) p_{t}}{2}\right] \leq\left[\frac{(n-4)\left(p_{t-1}+p_{t}\right)}{2}\right] .
$$

Let $H \in \operatorname{Ex}\left(p_{t-1}+p_{t}-n+1 ; K_{1, n-3}\right)$. As $p_{t-1}+p_{t}-n+1 \geq p_{t}+1 \geq n+1$, we have $e(H)=\left[\frac{(n-4)\left(p_{t-1}+p_{t}-n+1\right)}{2}\right]$ by Lemma 2.1. Clearly $K_{n-1} \cup H$ does not contain any copies of $T_{n}^{1}$ and

$$
\begin{aligned}
e\left(K_{n-1} \cup H\right) & =e\left(K_{n-1}\right)+e(H)=\left(\begin{array}{c}
n-1 \\
2
\end{array}\right)+\left[\frac{(n-4)\left(p_{t-1}+p_{t}-n+1\right)}{2}\right] \\
& =\left[\frac{(n-4)\left(p_{t-1}+p_{t}\right)}{2}\right]+n-1>e\left(G_{t-1} \cup G_{t}\right) .
\end{aligned}
$$

Since $G_{t-1} \cup G_{t} \in \operatorname{Ex}\left(p_{t-1}+p_{t} ; T_{n}^{1}\right)$, we get a contradiction. Hence $p_{1} \leq p_{2} \leq \cdots \leq p_{t-1} \leq$ $n-1$. Combining this with the previous assertion that $p_{t} \geq \cdots \geq p_{2} \geq n-1$ we obtain

$$
p_{1} \leq n-1, \quad p_{2}=\cdots=p_{t-1}=n-1 \quad \text { and } \quad p_{t} \geq n-1 .
$$

As $G$ is an extremal graph, we must have

$$
G_{1} \cong K_{p_{1}}, \quad G_{2} \cong K_{n-1}, \quad \ldots, \quad G_{t-1} \cong K_{n-1}
$$

If $p_{t}=n-1$, then $G_{t} \cong K_{n-1}$. By $(2.7), G \cong K_{p_{1}} \cup(t-1) K_{n-1} \cong k K_{n-1} \cup K_{r}$. Thus,

$$
e(G)=k\left(\begin{array}{c}
n-1 \\
2
\end{array}\right)+\left(\begin{array}{l}
r \\
2
\end{array}\right)=\frac{(n-2) p-r(n-1-r)}{2} \text { for } t \geq 2 \text { and } p_{t}=n-1 .
$$

Now we assume $p_{t} \geq n$. By Lemma $2.2, e\left(G_{t}\right)=\left[\frac{(n-4) p_{t}}{2}\right]$. Since $p_{1} \leq n-1$, we have $G_{1} \cong K_{p_{1}}$ and so $e\left(G_{1}\right)=e\left(K_{p_{1}}\right)=\left(\begin{array}{c}p_{1} \\ 2\end{array}\right)$. Let $H_{1} \in \operatorname{Ex}\left(p_{1}+p_{t} ; K_{1, n-3}\right)$. Then $H_{1}$ does not contain $T_{n}^{1}$ as a subgraph. By Lemma 2.1 , for $p_{1} \leq n-4$ we have

$$
\begin{aligned}
e\left(H_{1}\right) & =\left[\frac{(n-4)\left(p_{1}+p_{t}\right)}{2}\right] \geq\left[\frac{(n-4) p_{t}}{2}\right]+\left[\frac{(n-4) p_{1}}{2}\right] \\
& \geq\left[\frac{(n-4) p_{t}}{2}\right]+\frac{(n-4)\left(p_{1}-1\right)}{2}+1 \\
& >\left[\frac{(n-4) p_{t}}{2}\right]+\frac{p_{1}\left(p_{1}-1\right)}{2}=e\left(G_{1} \cup G_{t}\right) .
\end{aligned}
$$

This contradicts $G_{1} \cup G_{t} \in \operatorname{Ex}\left(p_{1}+p_{t} ; T_{n}^{1}\right)$. Hence $n-3 \leq p_{1} \leq n-1$.

For $p_{1} \in\{n-3, n-2\}$ and $p_{t} \geq n$, we have $p_{1}\left(p_{1}-(n-3)\right) \leq 2 n-4$ and so

$$
\begin{aligned}
e\left(G_{1} \cup G_{t}\right) & =e\left(G_{1}\right)+e\left(G_{t}\right)=\left(\begin{array}{c}
p_{1} \\
2
\end{array}\right)+\left[\frac{(n-4) p_{t}}{2}\right] \\
& \leq \frac{p_{1}\left(p_{1}-1\right)+(n-4) p_{t}}{2}=\frac{p_{1}\left(p_{1}-(n-3)\right)+(n-4)\left(p_{1}+p_{t}\right)}{2}
\end{aligned}
$$




$$
\begin{aligned}
& \leq \frac{2 n-4+(n-4)\left(p_{1}+p_{t}\right)}{2}=\left(\begin{array}{c}
n-1 \\
2
\end{array}\right)+\frac{(n-4)\left(p_{1}+p_{t}-n+1\right)-2}{2} \\
& <\left(\begin{array}{c}
n-1 \\
2
\end{array}\right)+\left[\frac{(n-4)\left(p_{1}+p_{t}-n+1\right)}{2}\right] .
\end{aligned}
$$

Let $H_{2} \in \operatorname{Ex}\left(p_{1}+p_{t}-n+1 ; K_{1, n-3}\right)$. Then $K_{n-1} \cup H_{2}$ does not contain any copies of $T_{n}^{1}$. Since $p_{1}+p_{t}-n+1 \geq p_{1}+1 \geq n-2$, applying Lemma 2.1 we have $e\left(H_{2}\right)=\left[\frac{(n-4)\left(p_{1}+p_{t}-n+1\right)}{2}\right]$. Thus, we have $e\left(K_{n-1} \cup H_{2}\right)=\left(\begin{array}{c}n-1 \\ 2\end{array}\right)+\left[\frac{(n-4)\left(p_{1}+p_{t}-n+1\right)}{2}\right]>e\left(G_{1} \cup G_{t}\right)$. This contradicts $G_{1} \cup G_{t} \in \operatorname{Ex}\left(p_{1}+p_{t} ; T_{n}^{1}\right)$.

By the above, for $t \geq 2$ and $p_{t} \geq n$ we have $p_{1}=p_{2}=\cdots=p_{t-1}=n-1$. If $p_{t} \geq 2 n-2$, setting $H_{3} \in \operatorname{Ex}\left(p_{t}-(n-1) ; K_{1, n-3}\right)$ and then applying Lemmas 2.1 and 2.2 we find that

$$
e\left(G_{t}\right)=\left[\frac{(n-4) p_{t}}{2}\right]<\left(\begin{array}{c}
n-1 \\
2
\end{array}\right)+\left[\frac{(n-4)\left(p_{t}-(n-1)\right)}{2}\right]=e\left(K_{n-1} \cup H_{3}\right) .
$$

This contradicts the fact $G_{t} \in \operatorname{Ex}\left(p_{t} ; T_{n}^{1}\right)$. Hence $n \leq p_{t}<2 n-2$ and so $r \geq 1$. Note that $p=k(n-1)+r=(k-1)(n-1)+n-1+r$ and $n \leq n-1+r<2 n-2$. Hence $t=k$, $p_{t}=n-1+r$ and therefore

$$
\begin{aligned}
e(G) & =e\left((k-1) K_{n-1}\right)+e\left(G_{t}\right)=(k-1)\left(\begin{array}{c}
n-1 \\
2
\end{array}\right)+\left[\frac{(n-4)(n-1+r)}{2}\right] \\
& =\left[\frac{(n-2) p}{2}\right]-(n-1+r) \quad \text { for } t \geq 2 \text { and } p_{t} \geq n .
\end{aligned}
$$

Since $G \in \operatorname{Ex}\left(p ; T_{n}^{1}\right)$, by comparing (2.5), (2.8) and (2.9) we get

$$
e(G)=\max \left\{\left[\frac{(n-4) p}{2}\right], \frac{(n-2) p-r(n-1-r)}{2},\left[\frac{(n-2) p}{2}\right]-(n-1+r)\right\} .
$$

Observe that $p=k(n-1)+r \geq n-1+r$. We see that $\left[\frac{(n-4) p}{2}\right]=\left[\frac{(n-2) p}{2}\right]-p \leq\left[\frac{(n-2) p}{2}\right]-$ $(n-1+r)$ and therefore

$$
\begin{aligned}
\operatorname{ex}\left(p ; T_{n}^{1}\right) & =e(G)=\max \left\{\frac{(n-2) p-r(n-1-r)}{2},\left[\frac{(n-2) p}{2}\right]-(n-1+r)\right\} \\
& =\frac{(n-2) p-r(n-1-r)}{2}+\max \left\{0,\left[\frac{r(n-3-r)-2(n-1)}{2}\right]\right\} .
\end{aligned}
$$

For $7 \leq n \leq 12$ we have $r(n-3-r)-2(n-1) \leq \frac{(n-3)^{2}}{4}-2(n-1)=\frac{(n-7)^{2}-32}{4}<0$. For $r \in\{0,1,2, n-5, n-4, n-3, n-2\}$ we see that $r(n-3-r)-2(n-1)<0$. Suppose $n \geq 13$ and $3 \leq r \leq n-6$. For $4 \leq r \leq n-7$ we have $\left|r-\frac{n-3}{2}\right| \leq \frac{n-11}{2}$ and so

$$
\begin{aligned}
r(n-3-r)-2(n-1) & =\frac{n^{2}-14 n+17}{4}-\left(r-\frac{n-3}{2}\right)^{2} \\
& \geq \frac{n^{2}-14 n+17}{4}-\left(\frac{n-11}{2}\right)^{2}=2 n-26 \geq 0 .
\end{aligned}
$$

For $r \in\{3, n-6\}$ we have $r(n-3-r)-2(n-1)=3(n-6)-2(n-1)=n-16$. Now combining the above with (2.10) we deduce the result. 
Corollary 2.1. Suppose $p, n \in \mathbb{N}, p \geq n \geq 5$ and $n-1 \nmid p$. Then $\frac{(n-2) p}{2}-\frac{(n-1)^{2}}{8} \leq$ $e x\left(p ; T_{n}^{1}\right) \leq \frac{(n-2)(p-1)}{2}$.

Proof. Suppose $p=k(n-1)+r$ with $k \in \mathbb{N}$ and $r \in\{0,1, \ldots, n-2\}$. Then $r \geq 1$. Clearly $\frac{(n-1)^{2}}{4} \geq r(n-1-r)=\left(\frac{n-1}{2}\right)^{2}-\left(\frac{n-1}{2}-r\right)^{2} \geq\left(\frac{n-1}{2}\right)^{2}-\left(\frac{n-1}{2}-1\right)^{2}=n-2$ and $n-1+r>\frac{n-2}{2}$. Thus, from Theorem 2.1 we deduce that $\operatorname{ex}\left(p ; T_{n}^{1}\right) \leq \frac{(n-2) p-(n-2)}{2}$ and $\operatorname{ex}\left(p ; T_{n}^{1}\right) \geq \frac{(n-2) p-r(n-1-r)}{2} \geq \frac{(n-2) p-(n-1)^{2} / 4}{2}$. This proves the corollary.

\section{Evaluation of $\operatorname{ex}\left(p ; T_{n}^{2}\right)$}

Lemma 3.1. Let $p, n \in \mathbb{N}, p \geq n \geq 7$ and $G \in E x\left(p ; T_{n}^{2}\right)$. Suppose that $G$ is connected. Then $\Delta(G) \leq n-3$. Moreover, for $p<2 n-2$ we have $\Delta(G) \leq n-4$.

Proof. Since a graph does not contain $K_{1, n-3}$ implies that the graph does not contain $T_{n}^{2}$, by Lemma 2.1 we have

$$
e(G)=\operatorname{ex}\left(p ; T_{n}^{2}\right) \geq \operatorname{ex}\left(p ; K_{1, n-3}\right)=\left[\frac{(n-4) p}{2}\right] .
$$

Suppose that $v_{0} \in V(G), d\left(v_{0}\right)=\Delta(G)=m$ and $\Gamma\left(v_{0}\right)=\left\{v_{1}, \ldots, v_{m}\right\}$. If $V(G)=$ $\left\{v_{0}, v_{1}, \ldots, v_{m}\right\}$, then $m=p-1 \geq n-1$. Since $G$ does not contain $T_{n}^{2}$, we see that $G\left[v_{1}, \ldots, v_{m}\right]$ does not contain $K_{1,2}$ and hence $e\left(G\left[v_{1}, \ldots, v_{m}\right]\right) \leq \frac{m}{2}$. Therefore $e(G)=$ $d\left(v_{0}\right)+e\left(G\left[v_{1}, \ldots, v_{m}\right]\right) \leq m+\frac{m}{2}=\frac{3(p-1)}{2} \leq \frac{(n-4) p-3}{2}<\left[\frac{(n-4) p}{2}\right]$. This contradicts $(3.1)$. Thus $p>m+1$. Suppose that $u_{1}, \ldots, u_{t}$ are all vertices such that $d\left(u_{1}, v_{0}\right)=\cdots=$ $d\left(u_{t}, v_{0}\right)=2$. Then $t \geq 1$. We may assume without loss of generality that $v_{1}, \ldots, v_{s}$ are all vertices in $\Gamma\left(v_{0}\right)$ adjacent to some vertex in the set $\left\{u_{1}, \ldots, u_{t}\right\}$. Then $1 \leq s \leq m$. Let $V_{1}=\left\{v_{0}, v_{1}, \ldots, v_{m}\right\}, V_{1}^{\prime}=V(G)-V_{1}$ and let $e\left(V_{1} V_{1}^{\prime}\right)$ be the number of edges with one endpoint in $V_{1}$ and another endpoint in $V_{1}^{\prime}$. Since $G$ does not contain $T_{n}^{2}$, for $m \geq n-3$ each $v_{i}(1 \leq i \leq s)$ has one and only one adjacent vertex in the set $\left\{u_{1}, \ldots, u_{t}\right\}$. Thus, for $m \geq n-3$ we must have $e\left(V_{1} V_{1}^{\prime}\right)=s \geq t$.

If $m \geq n-1$, since $G$ does not contain $T_{n}^{2}$ as a subgraph, we see that $d\left(v_{i}\right) \leq 2$ for $i=1, \ldots, m$ and so $e\left(G\left[V_{1}\right]\right)=d\left(v_{0}\right)+e\left(G\left[v_{s+1}, \ldots, v_{m}\right]\right) \leq m+\frac{m-s}{2}$. Hence

$$
\begin{aligned}
e(G) & =e\left(G\left[V_{1}\right]\right)+e\left(V_{1} V_{1}^{\prime}\right)+e\left(G-V_{1}\right) \\
& \leq \frac{3 m-s}{2}+s+e\left(G-V_{1}\right) \leq 2 m+e\left(G-V_{1}\right) .
\end{aligned}
$$

Suppose $m+1=k(n-1)+r$ with $k \in \mathbb{N}$ and $0 \leq r \leq n-2$. Set $G_{1}=k K_{n-1} \cup K_{r}$. Since $m+1 \geq n$, by $(2.2)$ we have $e\left(G_{1}\right)>2(m+1)-1>2 m$. Thus, $e\left(G_{1} \cup\left(G-V_{1}\right)\right)=$ $e\left(G_{1}\right)+e\left(G-V_{1}\right)>2 m+e\left(G-V_{1}\right) \geq e(G)$. As $G_{1}$ does not contain any copies of $T_{n}^{2}$ and $G$ is an extremal graph, we get a contradiction. Hence $\Delta(G)=m \leq n-2$.

Suppose $m=n-2$. As $G$ does not contain $T_{n}^{2}$ as a subgraph, we see that $d\left(v_{1}\right)=\cdots=$ 
$d\left(v_{s}\right)=2$ and so $e\left(G\left[V_{1}\right]\right) \leq n-2+\left(\begin{array}{c}n-2-s \\ 2\end{array}\right)$. Since $1 \leq s \leq m=n-2 \leq 2 n-8$, we have

$$
\begin{aligned}
e(G) & =e\left(G\left[V_{1}\right]\right)+e\left(V_{1} V_{1}^{\prime}\right)+e\left(G-V_{1}\right) \\
& \leq\left(\begin{array}{c}
n-2-s \\
2
\end{array}\right)+n-2+s+e\left(G-V_{1}\right) \\
& =\frac{(n-2)(n-1)-s(2 n-7-s)}{2}+e\left(G-V_{1}\right) \\
& <\left(\begin{array}{c}
n-1 \\
2
\end{array}\right)+e\left(G-V_{1}\right)=e\left(K_{n-1} \cup\left(G-V_{1}\right)\right) .
\end{aligned}
$$

This is impossible since $G$ is an extremal graph.

By the above, $\Delta(G) \leq n-3$. We first assume $\Delta(G)=n-3$. We claim that $d\left(v_{i}\right) \leq n-4$ for $i=1,2, \ldots, s$. If $i \in\{1,2, \ldots, s\}$ and $d\left(v_{i}\right)=n-3$, let $u_{j}$ be the unique adjacent vertex of $v_{i}$ in $\left\{u_{1}, \ldots, u_{t}\right\}$ and let $V_{2}=\left\{v_{0}, v_{1}, \ldots, v_{n-3}, u_{j}\right\}$. Then there is at most one vertex adjacent to $u_{j}$ in $G-V_{2}$. Hence $e\left(G-V_{1}\right) \leq 1+e\left(G-V_{2}\right)$. Since each $v_{r}(1 \leq r \leq s)$ is adjacent to one and only one vertex in $\left\{u_{1}, \ldots, u_{t}\right\}$ and $\Delta\left(G\left[V_{1}\right]\right) \leq n-3$, we see that

$$
e\left(G\left[V_{1}\right]\right)=\frac{1}{2} \sum_{r=0}^{n-3} d_{G\left[V_{1}\right]}\left(v_{r}\right) \leq \frac{s(n-4)+(n-2-s)(n-3)}{2}=\frac{(n-2)(n-3)-s}{2} .
$$

Note that $s \leq \Delta(G)=n-3$. From the above we deduce that

$$
\begin{aligned}
e(G) & =e\left(G\left[V_{1}\right]\right)+e\left(V_{1} V_{1}^{\prime}\right)+e\left(G-V_{1}\right)=e\left(G\left[V_{1}\right]\right)+s+e\left(G-V_{1}\right) \\
& \leq e\left(G\left[V_{1}\right]\right)+s+1+e\left(G-V_{2}\right) \leq \frac{(n-2)(n-3)-s}{2}+s+1+e\left(G-V_{2}\right) \\
& =\frac{(n-2)(n-3)+s+2}{2}+e\left(G-V_{2}\right) \leq \frac{(n-2)(n-3)+n-1}{2}+e\left(G-V_{2}\right) \\
& <\frac{(n-1)(n-2)}{2}+e\left(G-V_{2}\right)=e\left(K_{n-1} \cup\left(G-V_{2}\right)\right) .
\end{aligned}
$$

Since $K_{n-1} \cup\left(G-V_{2}\right)$ does not contain $T_{n}^{2}$ and $G$ is an extremal graph, we get a contradiction. Hence the claim is true. Thus, for $\Delta(G)=n-3$ we have $d_{G\left[V_{1}\right]}\left(v_{i}\right) \leq n-5$ for $i=1,2, \ldots, s$ and so

$$
e\left(G\left[V_{1}\right]\right)=\frac{1}{2} \sum_{i=0}^{n-3} d_{G\left[V_{1}\right]}\left(v_{i}\right) \leq \frac{s(n-5)+(n-2-s)(n-3)}{2}=\frac{(n-2)(n-3)}{2}-s .
$$

Now we assume $p<2 n-2$ and $p=n-1+r$. Then $1 \leq r<n-1$. By the above, $\Delta(G) \leq n-3$. Assume $\Delta(G)=n-3$. Then $\left|V\left(G-V_{1}\right)\right|=p-(n-2)=r+1<n$, $\Delta\left(G-V_{1}\right) \leq n-3$ and so $e\left(G-V_{1}\right) \leq \min \left\{\left(\begin{array}{c}r+1 \\ 2\end{array}\right), \frac{(r+1)(n-3)}{2}\right\}$. Since $e\left(G\left[V_{1}\right]\right) \leq \frac{(n-2)(n-3)}{2}-s$ by (3.2), we deduce that

$$
\begin{aligned}
& e(G)=e\left(G\left[V_{1}\right]\right)+e\left(V_{1} V_{1}^{\prime}\right)+e\left(G-V_{1}\right) \\
& \leq \frac{(n-2)(n-3)}{2}-s+s+\min \left\{\frac{r(r+1)}{2}, \frac{(r+1)(n-3)}{2}\right\} \\
& = \begin{cases}\frac{(n-2)(n-3)}{2}+\left(\begin{array}{c}
r+1 \\
2
\end{array}\right) & \text { if } \quad r \leq n-3 \\
\frac{(n-2)(n-3)}{2}+\frac{(n-3)(n-1)}{2} & \text { if } \quad r=n-2\end{cases} \\
& <\left(\begin{array}{c}
n-1 \\
2
\end{array}\right)+\left(\begin{array}{l}
r \\
2
\end{array}\right)=e\left(K_{n-1} \cup K_{r}\right) .
\end{aligned}
$$


This is impossible since $G$ is an extremal graph. Thus, $\Delta(G) \leq n-4$ for $p<2 n-2$. Now the proof is complete.

Lemma 3.2. Let $p, n \in \mathbb{N}, p \geq n \geq 7$ and $G \in E x\left(p ; T_{n}^{2}\right)$. Suppose that $G$ is connected. Then $p<2 n-2$.

Proof. By Lemma 3.1, we have $\Delta(G) \leq n-3$ and so $e(G) \leq \frac{(n-3) p}{2}$. Assume that $p=k(n-1)+r$ with $k \in \mathbb{N}$ and $r \in\{0,1, \ldots, n-2\}$. Let $G_{1} \in \operatorname{Ex}\left(n-1+r ; K_{1, n-3}\right)$. Then $e\left(G_{1}\right)=\left[\frac{(n-4)(n-1+r)}{2}\right]$ by Lemma 2.1. Hence, if $(k-2)(n-1)-r \geq 2$, then

$$
\begin{aligned}
e\left((k-1) K_{n-1} \cup G_{1}\right) & =(k-1)\left(\begin{array}{c}
n-1 \\
2
\end{array}\right)+\left[\frac{(n-4)(n-1+r)}{2}\right] \\
& =\frac{(p-r-(n-1))(n-2)}{2}+\left[\frac{(n-4)(n-1+r)}{2}\right] \\
& =\left[\frac{(n-3) p}{2}+\frac{p-2 r-2(n-1)}{2}\right] \\
& =\left[\frac{(n-3) p}{2}+\frac{(k-2)(n-1)-r}{2}\right]>\left[\frac{(n-3) p}{2}\right] \geq e(G) .
\end{aligned}
$$

This is impossible since $(k-1) K_{n-1} \cup G_{1}$ does not contain $T_{n}^{2}$ as a subgraph and $G \in$ $\operatorname{Ex}\left(p ; T_{n}^{2}\right)$. Thus $(k-2)(n-1)-r \leq 1$. If $k=3$, then $r=n-2$ and $p=3(n-1)+n-2=4 n-5$ and so

$$
\begin{aligned}
e(G) & \leq\left[\frac{(n-3) p}{2}\right] \leq \frac{(n-3)(4 n-5)}{2}=\frac{4 n^{2}-17 n+15}{2} \\
& <\frac{4 n^{2}-14 n+12}{2}=3\left(\begin{array}{c}
n-1 \\
2
\end{array}\right)+\left(\begin{array}{c}
n-2 \\
2
\end{array}\right)=e\left(3 K_{n-1} \cup K_{n-2}\right) .
\end{aligned}
$$

Since $3 K_{n-1} \cup K_{n-2}$ does not contain $T_{n}^{2}$ and $G \in \operatorname{Ex}\left(p ; T_{n}^{2}\right)$, we get a contradiction. Thus $k \leq 2$.

For $p=2(n-1)+r$ with $r \in\{0,1,2, n-4, n-3, n-2\}$ we see that $r(n-2-r)<2 n-2$ and so $e\left(2 K_{n-1} \cup K_{r}\right)=\frac{2(n-1)(n-2)+r(r-1)}{2}>\frac{(n-3)(2 n-2+r)}{2} \geq e(G)$. This contradicts the assumption $G \in \operatorname{Ex}\left(p ; T_{n}^{2}\right)$. Now suppose $p=2(n-1)+r$ with $3 \leq r \leq n-5$. If $\Delta(G) \leq n-4$, then $e(G) \leq \frac{(n-4) p}{2}$. From previous argument we have

$$
\begin{aligned}
e\left(K_{n-1} \cup G_{1}\right) & =\left(\begin{array}{c}
n-1 \\
2
\end{array}\right)+\left[\frac{(n-4)(n-1+r)}{2}\right]=\left[\frac{(n-3) p-r}{2}\right] \\
& =\left[\frac{(n-4) p}{2}\right]+n-1>\frac{(n-4) p}{2} \geq e(G) .
\end{aligned}
$$

Since $K_{n-1} \cup G_{1}$ does not contain $T_{n}^{2}$ as a subgraph and $G \in \operatorname{Ex}\left(p ; T_{n}^{2}\right)$, we get a contradiction. Hence $\Delta(G)=n-3$. Suppose $v_{0} \in V(G), d\left(v_{0}\right)=n-3, \Gamma\left(v_{0}\right)=\left\{v_{1}, \ldots, v_{n-3}\right\}, V_{1}=$ $\left\{v_{0}, v_{1}, \ldots, v_{n-3}\right\}$ and $V_{1}^{\prime}=V(G)-V_{1}$. Suppose also that there are exactly $s$ vertices in $\Gamma\left(v_{0}\right)$ adjacent to some vertex in $V_{1}^{\prime}$. Then $1 \leq s \leq n-3$. By $(3.2), e\left(G\left[V_{1}\right]\right) \leq \frac{(n-2)(n-3)}{2}-s$. As $G$ does not contain any copies of $T_{n}^{2}$, we see that $e\left(V_{1} V_{1}^{\prime}\right)=s$. Since $\left|V\left(G-V_{1}\right)\right|=$ $\left|V_{1}^{\prime}\right|=p-(n-2)=n+r$ and $G-V_{1}$ does not contain any copies of $T_{n}^{2}$ we see that $e\left(G-V_{1}\right) \leq \operatorname{ex}\left(n+r ; T_{n}^{2}\right)$.

We claim that

$$
\operatorname{ex}\left(n+r ; T_{n}^{2}\right) \leq \max \left\{\frac{(n-4)(n+r)}{2}, \frac{(n-1)(n-2)+r(r+1)}{2}\right\}
$$


for $3 \leq r \leq n-5$. Let $G^{\prime} \in \operatorname{Ex}\left(n+r ; T_{n}^{2}\right)$. If $G^{\prime}$ is connected, using Lemma 3.1 we have $\Delta\left(G^{\prime}\right) \leq n-4$ and so $e\left(G^{\prime}\right) \leq \frac{(n-4)(n+r)}{2}$. Now suppose that $G^{\prime}$ is not connected. If $n_{1}, n_{2} \in\{1,2, \ldots, n-2\}$, from Lemma 2.3 we have $e\left(K_{n_{1}} \cup K_{n_{2}}\right)<e\left(K_{n_{1}+n_{2}}\right)$ for $n_{1}+n_{2}<n$ and $e\left(K_{n_{1}} \cup K_{n_{2}}\right)<e\left(K_{n-1} \cup K_{n_{1}+n_{2}-(n-1)}\right)$ for $n_{1}+n_{2} \geq n$. Thus, $G^{\prime}=G_{1}^{\prime} \cup G_{2}^{\prime}$, where $G_{1}^{\prime}$ and $G_{2}^{\prime}$ are components of $G^{\prime}$ with $\left|V\left(G_{1}^{\prime}\right)\right|=p_{1}^{\prime}<n-1$ and $\left|V\left(G_{2}^{\prime}\right)\right|=p_{2}^{\prime} \geq n-1$. For $p_{2}^{\prime} \geq n$ we have $p_{1}^{\prime} \leq r \leq n-3$ and so $e\left(G_{1}^{\prime}\right)=\frac{p_{1}^{\prime}\left(p_{1}^{\prime}-1\right)}{2} \leq \frac{(n-4) p_{1}^{\prime}}{2}$. For $p_{2}^{\prime} \geq n$ we also have $\Delta\left(G_{2}^{\prime}\right) \leq n-4$ and so $e\left(G_{2}^{\prime}\right) \leq \frac{(n-4) p_{2}^{\prime}}{2}$ by Lemma 3.1. Hence for $p_{2}^{\prime} \geq n$ we find that $e\left(G^{\prime}\right)=e\left(G_{1}^{\prime}\right)+e\left(G_{2}^{\prime}\right) \leq \frac{(n-4) p_{1}^{\prime}}{2}+\frac{(n-4) p_{2}^{\prime}}{2}=\frac{(n-4)(n+r)}{2}$. Now assume $p_{2}^{\prime}=n-1$. Then $p_{1}^{\prime}=r+1$ and

$$
e\left(G^{\prime}\right)=e\left(K_{n-1} \cup K_{r+1}\right)=\frac{(n-1)(n-2)+r(r+1)}{2} .
$$

Hence the claim is true and so

$$
e\left(G-V_{1}\right) \leq \operatorname{ex}\left(n+r ; T_{n}^{2}\right) \leq \max \left\{\frac{(n-4)(n+r)}{2}, \frac{(n-1)(n-2)+r(r+1)}{2}\right\} .
$$

Thus,

$$
\begin{aligned}
e(G) & =e\left(G\left[V_{1}\right]\right)+e\left(V_{1} V_{1}^{\prime}\right)+e\left(G-V_{1}\right) \\
& \leq \frac{(n-2)(n-3)}{2}-s+s+\max \left\{\frac{(n-4)(n+r)}{2}, \frac{(n-1)(n-2)+r(r+1)}{2}\right\} \\
& =\left(\begin{array}{c}
n-1 \\
2
\end{array}\right)+\max \left\{\frac{(n-4)(n-1+r)-n}{2}, \frac{(n-1)(n-2)+r(r-1)}{2}-(n-2-r)\right\} \\
& <\left(\begin{array}{c}
n-1 \\
2
\end{array}\right)+\max \left\{\left[\frac{(n-4)(n-1+r)}{2}\right], \frac{(n-1)(n-2)+r(r-1)}{2}\right\} \\
& =\max \left\{e\left(K_{n-1} \cup G_{1}\right), e\left(2 K_{n-1} \cup K_{r}\right)\right\} .
\end{aligned}
$$

This is impossible since $G$ is an extremal graph.

By the above we must have $k=1$ and so $p=k(n-1)+r<2 n-2$ as asserted.

Lemma 3.3. Let $p, n \in \mathbb{N}, p \geq n \geq 7$ and $G \in E x\left(p ; T_{n}^{2}\right)$. Suppose that $G$ is connected. Then $\Delta(G)=n-4$ and $e(G)=\left[\frac{(n-4) p}{2}\right]$.

Proof. By (3.1), $e(G) \geq\left[\frac{(n-4) p}{2}\right]$. If $\Delta(G) \leq n-5$, using Euler's theorem we see that $e(G)=\frac{1}{2} \sum_{v \in V(G)} d(v) \leq \frac{(n-5) p}{2}$. Hence $\frac{(n-4) p-1}{2} \leq\left[\frac{(n-4) p}{2}\right] \leq e(G) \leq \frac{(n-5) p}{2}$. This is impossible. Thus $\Delta(G) \geq n-4$. By Lemmas 3.1 and 3.2, $\Delta(G) \leq n-4$. Therefore $\Delta(G)=n-4$ and so $e(G)=\frac{1}{2} \sum_{v \in V(G)} d(v) \leq \frac{(n-4) p}{2}$. Recall that $e(G) \geq\left[\frac{(n-4) p}{2}\right]$. Then $e(G)=\left[\frac{(n-4) p}{2}\right]$ as asserted.

Lemma 3.4. Let $p$ and $k$ be nonnegative integers, $p=5 k+r$ and $r \in\{0,1,2,3,4\}$. Suppose that $G$ is a graph of order $p$ without $T_{6}^{2}$. Then $e(G) \leq 2 p-\frac{r(5-r)}{2}$.

Proof. Clearly $\Delta\left(T_{6}^{2}\right)=3$. We prove the lemma by induction on $p$. For $p \leq 5$ we have $e(G) \leq \frac{p(p-1)}{2}=2 p-\frac{r(5-r)}{2}$. Now suppose that $p \geq 6$ and the lemma is true for all graphs of order $p_{0}<p$ without $T_{6}^{2}$. If $\Delta(G) \leq 3$, then $e(G)=\frac{1}{2} \sum_{v \in V(G)} d(v) \leq \frac{3 p}{2} \leq 2 p-3 \leq$ $2 p-\frac{r(5-r)}{2}$.

Suppose $\Delta(G)=m \geq 4, v_{0} \in V(G), d\left(v_{0}\right)=m, \Gamma\left(v_{0}\right)=\left\{v_{1}, \ldots, v_{m}\right\}, V_{1}=\left\{v_{0}, v_{1}, \ldots, v_{m}\right\}$ and $V_{1}^{\prime}=V(G)-V_{1}$. If $G\left[V_{1}\right]$ is a component of $G$, then $e\left(G\left[V_{1}\right]\right)=e\left(K_{5}\right)=10$ for $m=4$, 
and $e\left(G\left[V_{1}\right]\right) \leq m+\frac{m}{2}=\frac{3 m}{2}$ for $m \geq 5$ since $d\left(v_{i}\right) \leq 2$ for $i=1,2, \ldots, m$. By the inductive hypothesis, $e\left(G\left[V_{1}^{\prime}\right]\right) \leq 2(p-m-1)-\frac{r_{1}\left(5-r_{1}\right)}{2}$, where $r_{1} \in\{0,1,2,3,4\}$ is given by $p-m-1 \equiv r_{1}(\bmod 5)$. Thus, for $m=4$ we have $e(G)=e\left(G\left[V_{1}\right]\right)+e\left(G\left[V_{1}^{\prime}\right]\right) \leq$ $10+2(p-5)-\frac{r(5-r)}{2}=2 p-\frac{r(5-r)}{2}$, and for $m \geq 5$ we have $e(G)=e\left(G\left[V_{1}\right]\right)+e\left(G\left[V_{1}^{\prime}\right]\right) \leq$ $\frac{3 m}{2}+2(p-m-1)-\frac{r_{1}\left(5-r_{1}\right)}{2} \leq 2 p-2-\frac{m}{2} \leq 2 p-3 \leq 2 p-\frac{r(5-r)}{2}$.

From now on we assume that $G\left[V_{1}\right]$ is not a component of $G$ and $m=\Delta(G) \geq 4$. Hence there is a vertex $u_{1}$ such that $d\left(u_{1}, v_{0}\right)=2$ and $u_{1} v_{1} \in E(G)$ with no loss of generality. Then $v_{1} v_{i} \notin E(G)$ for $i=2,3, \ldots, m$. For $m=4$ we see that $e\left(G\left[V_{1}\right]\right)+e\left(V_{1} V_{1}^{\prime}\right) \leq 4+4=8$. For $m \geq 5$ we see that $d\left(v_{i}\right) \leq 2$ for $i=1,2, \ldots, m$ and so $e\left(G\left[V_{1}\right]\right)+e\left(V_{1} V_{1}^{\prime}\right) \leq \sum_{i=1}^{m} d\left(v_{i}\right) \leq 2 m$. Hence, for $m \geq 4$ we have $e(G)=e\left(G\left[V_{1}\right]\right)+e\left(V_{1} V_{1}^{\prime}\right)+e\left(G\left[V_{1}^{\prime}\right]\right) \leq 2 m+e\left(G\left[V_{1}^{\prime}\right]\right)$. By the inductive hypothesis, $e\left(G\left[V_{1}^{\prime}\right]\right) \leq 2(p-m-1)-\frac{r_{1}\left(5-r_{1}\right)}{2}$, where $r_{1} \in\{0,1,2,3,4\}$ is given by $p-m-1 \equiv r_{1}(\bmod 5)$. Thus, $e(G) \leq 2 m+2(p-m-1)-\frac{r_{1}\left(5-r_{1}\right)}{2}=2 p-2-\frac{r_{1}\left(5-r_{1}\right)}{2}$. For $r_{1} \geq 1$ we have $e(G) \leq 2 p-2-2<2 p-\frac{r(5-r)}{2}$. For $r_{1}=0$ and $r=0,1,4$ we have $e(G) \leq$ $2 p-2 \leq 2 p-\frac{r(5-r)}{2}$. Therefore, we only need to consider the case $p \equiv m+1 \equiv 2,3(\bmod 5)$.

Now assume $p \equiv m+1 \equiv 2,3(\bmod 5)$ and $\Gamma\left(u_{1}\right)-\left\{v_{1}, \ldots, v_{m}\right\}=\left\{w_{1}, \ldots, w_{t}\right\}$. As $m \geq 4$ we have $m \geq 6$. Set $V_{2}=\left\{v_{0}, v_{1}, \ldots, v_{m}, u_{1}\right\}$ and $V_{2}^{\prime}=V(G)-V_{2}$. Since $d\left(v_{i}\right) \leq 2$ for $i=1,2, \ldots, m$, we see that

$$
e(G)=e\left(G\left[V_{2}\right]\right)+e\left(V_{2} V_{2}^{\prime}\right)+e\left(G\left[V_{2}^{\prime}\right]\right) \leq \sum_{i=1}^{m} d\left(v_{i}\right)+t+e\left(G\left[V_{2}^{\prime}\right]\right) \leq 2 m+t+e\left(G\left[V_{2}^{\prime}\right]\right) .
$$

Note that $p-m-2 \equiv 4(\bmod 5)$ and $e\left(G\left[V_{2}^{\prime}\right]\right) \leq 2(p-m-2)-\frac{4(5-4)}{2}$ by the inductive hypothesis. We then have $e(G) \leq 2 m+t+2(p-m-2)-2=2 p+t-6$. For $t \leq 3$ we get $e(G) \leq 2 p+t-6 \leq 2 p-3=2 p-\frac{r(5-r)}{2}$. For $t \geq 4$ set $V_{3}=\left\{v_{0}, v_{1}, \ldots, v_{m}, u_{1}, w_{1}, \ldots, w_{t}\right\}$ and $V_{3}^{\prime}=V(G)-V_{3}$. Since $d\left(v_{i}\right) \leq 2$ for $i=1,2, \ldots, m$ and $d\left(w_{j}\right) \leq 2$ for $j=1,2, \ldots, t$, using the inductive hypothesis we see that

$$
\begin{aligned}
e(G) & =e\left(G\left[V_{3}\right]\right)+e\left(V_{3} V_{3}^{\prime}\right)+e\left(G\left[V_{3}^{\prime}\right]\right) \leq \sum_{i=1}^{m} d\left(v_{i}\right)+\sum_{j=1}^{t} d\left(w_{j}\right)+e\left(G\left[V_{3}^{\prime}\right]\right) \\
& \leq 2 m+2 t+e\left(G\left[V_{3}^{\prime}\right]\right) \leq 2 m+2 t+2(p-m-2-t)=2 p-4 \\
& <2 p-\frac{r(5-r)}{2}
\end{aligned}
$$

By the above, the lemma has been proved by induction.

Theorem 3.1. Let $p, n \in \mathbb{N}, p \geq n-1 \geq 4$ and $p=k(n-1)+r$, where $k \in \mathbb{N}$ and $r \in\{0,1, \ldots, n-2\}$. Then

$$
\begin{aligned}
\operatorname{ex}\left(p ; T_{n}^{2}\right)= & \max \left\{\left[\frac{(n-2) p}{2}\right]-(n-1+r), \frac{(n-2) p-r(n-1-r)}{2}\right\} \\
= & \begin{cases}{\left[\frac{(n-2) p}{2}\right]-(n-1+r)} & \text { if } n \geq 16 \text { and } 3 \leq r \leq n-6 \text { or if } \\
\frac{(n-2) p-r(n-1-r)}{2} & 13 \leq n \leq 15 \text { and } 4 \leq r \leq n-7, \\
\text { otherwise. }\end{cases}
\end{aligned}
$$

Proof. Clearly ex $\left(n-1 ; T_{n}^{2}\right)=e\left(K_{n-1}\right)=\frac{(n-2)(n-1)}{2}$. Thus the result is true for $p=n-1$. Now we assume $p \geq n$. Since $T_{5}^{2} \cong T_{5}^{\prime}$, taking $n=5$ in [10, Theorem 3.1] we obtain the result 
in the case $n=5$. For $n=6$ we see that $\operatorname{ex}\left(p ; T_{6}^{2}\right) \geq e\left(k K_{5} \cup K_{r}\right)=10 k+\frac{r(r-1)}{2}=2 p-\frac{r(5-r)}{2}$. This together with Lemma 3.4 gives the result in this case. Applying Lemmas 3.3, 2.3 and replacing $T_{n}^{1}$ with $T_{n}^{2}$ in the proof of Theorem 2.1 we deduce the result for $n \geq 7$.

Corollary 3.1. Suppose $p, n \in \mathbb{N}, p \geq n \geq 5$ and $n-1 \nmid p$. Then $\frac{(n-2) p}{2}-\frac{(n-1)^{2}}{8} \leq$ $e x\left(p ; T_{n}^{2}\right) \leq \frac{(n-2)(p-1)}{2}$.

\section{The Ramsey number $r\left(T_{n}^{i}, T_{n}\right)$}

Lemma 4.1 ([9, Lemma 2.1]). Let $G_{1}$ and $G_{2}$ be two graphs. Suppose $p \in \mathbb{N}, p \geq$ $\max \left\{\left|V\left(G_{1}\right)\right|,\left|V\left(G_{2}\right)\right|\right\}$ and $\operatorname{ex}\left(p ; G_{1}\right)+\operatorname{ex}\left(p ; G_{2}\right)<\left(\begin{array}{l}p \\ 2\end{array}\right)$. Then $r\left(G_{1}, G_{2}\right) \leq p$.

Proof. Let $G$ be a graph of order $p$. If $e(G) \leq \operatorname{ex}\left(p ; G_{1}\right)$ and $e(\bar{G}) \leq \operatorname{ex}\left(p ; G_{2}\right)$, then $\operatorname{ex}\left(p ; G_{1}\right)+\operatorname{ex}\left(p ; G_{2}\right) \geq e(G)+e(\bar{G})=\left(\begin{array}{l}p \\ 2\end{array}\right)$. This contradicts the assumption. Hence, either $e(G)>\operatorname{ex}\left(p ; G_{1}\right)$ or $e(\bar{G})>\operatorname{ex}\left(p ; G_{2}\right)$. Therefore, $G$ contains a copy of $G_{1}$ or $\bar{G}$ contains a copy of $G_{2}$. This shows that $r\left(G_{1}, G_{2}\right) \leq|V(G)|=p$. So the lemma is proved.

Lemma $4.2\left(\left[9\right.\right.$, Lemma 2.3]). Let $G_{1}$ and $G_{2}$ be two graphs with $\Delta\left(G_{1}\right)=d_{1} \geq 2$ and $\Delta\left(G_{2}\right)=d_{2} \geq 2$. Then

(i) $r\left(G_{1}, G_{2}\right) \geq d_{1}+d_{2}-\left(1-(-1)^{\left(d_{1}-1\right)\left(d_{2}-1\right)}\right) / 2$.

(ii) Suppose that $G_{1}$ is a connected graph of order $m$ and $d_{1}<d_{2} \leq m$. Then $r\left(G_{1}, G_{2}\right) \geq$ $2 d_{2}-1 \geq d_{1}+d_{2}$.

(iii) If $G_{1}$ is a connected graph of order $m, d_{1} \neq m-1$ and $d_{2}>m$, then $r\left(G_{1}, G_{2}\right) \geq$ $d_{1}+d_{2}$.

Theorem 4.1. Let $n \in \mathbb{N}$ and $i, j \in\{1,2\}$.

(i) If $n$ is odd with $n \geq 17$, then $r\left(T_{n}^{i}, T_{n}^{j}\right)=2 n-7$.

(ii) If $n$ is even with $n \geq 12$, then $r\left(T_{n}^{i}, T_{n}^{j}\right)=2 n-6$.

Proof. Suppose $n \geq 12$. Since $\Delta\left(T_{n}^{i}\right)=\Delta\left(T_{n}^{j}\right)=n-3$, from Lemma 4.2 we know that $r\left(T_{n}^{i}, T_{n}^{j}\right) \geq 2 n-7$ for odd $n$, and $r\left(T_{n}^{i}, T_{n}^{j}\right) \geq 2 n-6$ for even $n$. If $n$ is odd with $n \geq 17$, using Theorems 2.1 and 3.1 (with $k=1$ and $r=n-6$ ) we see that

$$
\operatorname{ex}\left(2 n-7 ; T_{n}^{i}\right)=\frac{(n-2)(2 n-7)-1}{2}-(2 n-7)<\frac{(n-4)(2 n-7)}{2}=\frac{1}{2}\left(\begin{array}{c}
2 n-7 \\
2
\end{array}\right)
$$

and so $\operatorname{ex}\left(2 n-7 ; T_{n}^{i}\right)+\operatorname{ex}\left(2 n-7 ; T_{n}^{j}\right)<\left(\begin{array}{c}2 n-7 \\ 2\end{array}\right)$. Thus, by Lemma 4.1 we have $r\left(T_{n}^{i}, T_{n}^{j}\right) \leq$ $2 n-7$. Hence (i) is true. From Theorems 2.1 and 3.1 (with $k=1$ and $r=n-5$ ) we see that for $n \geq 12$,

$$
\begin{aligned}
\operatorname{ex}\left(2 n-6 ; T_{n}^{i}\right) & =\frac{(n-2)(2 n-6)-4(n-5)}{2}=n^{2}-7 n+16 \\
& <n^{2}-\frac{13}{2} n+\frac{21}{2}=\frac{1}{2}\left(\begin{array}{c}
2 n-6 \\
2
\end{array}\right)
\end{aligned}
$$

and so $\operatorname{ex}\left(2 n-6 ; T_{n}^{i}\right)+\operatorname{ex}\left(2 n-6 ; T_{n}^{j}\right)<\left(\begin{array}{c}2 n-6 \\ 2\end{array}\right)$. Thus, by Lemma 4.1 we have $r\left(T_{n}^{i}, T_{n}^{j}\right) \leq$ $2 n-6$. Hence $r\left(T_{n}^{i}, T_{n}^{j}\right)=2 n-6$ for even $n$, proving (ii).

Lemma 4.3. Let $n \in \mathbb{N}, n \geq 5$ and $i \in\{1,2\}$. Let $G_{n}$ be a connected graph of order $n$ such that ex $\left(2 n-5 ; G_{n}\right)<n^{2}-5 n+4$. Then $r\left(T_{n}^{i}, G_{n}\right) \leq 2 n-5$.

Proof. By Theorems 2.1 and 3.1, $\operatorname{ex}\left(2 n-5 ; T_{n}^{i}\right)=\frac{(n-2)(2 n-5)-3(n-4)}{2}=n^{2}-6 n+11$. Thus,

$$
\operatorname{ex}\left(2 n-5 ; G_{n}\right)+\operatorname{ex}\left(2 n-5 ; T_{n}^{i}\right)<n^{2}-5 n+4+n^{2}-6 n+11=\left(\begin{array}{c}
2 n-5 \\
2
\end{array}\right) .
$$


Appealing to Lemma 4.1 we obtain $r\left(T_{n}^{i}, G_{n}\right) \leq 2 n-5$.

Lemma 4.4 ([10, Theorem 3.1]). Let $p, n \in \mathbb{N}$ with $p \geq n \geq 5$. Let $r \in\{0,1, \ldots, n-2\}$ be given by $p \equiv r(\bmod n-1)$. Then

$$
\operatorname{ex}\left(p ; T_{n}^{\prime}\right)= \begin{cases}{\left[\frac{(n-2)(p-1)-r-1}{2}\right]} & \text { if } n \geq 7 \text { and } 2 \leq r \leq n-4, \\ \frac{(n-2) p-r(n-1-r)}{2} & \text { otherwise. }\end{cases}
$$

Theorem 4.2. Let $n \in \mathbb{N}, n \geq 8$ and $i \in\{1,2\}$. Then $r\left(T_{n}^{i}, T_{n}^{\prime}\right)=r\left(T_{n}^{i}, T_{n}^{*}\right)=2 n-5$.

Proof. Let $T_{n} \in\left\{T_{n}^{\prime}, T_{n}^{*}\right)$. As $2 K_{n-3}$ does not contain any copies of $T_{n}^{i}$ and $\overline{2 K_{n-3}}=$ $K_{n-3, n-3}$ does not contain any copies of $T_{n}$, we see that $r\left(T_{n}^{i}, T_{n}\right) \geq 1+2(n-3)=2 n-5$. Taking $p=2 n-5$ and $r=n-4$ in Lemma 4.4 we find that

$$
\operatorname{ex}\left(2 n-5 ; T_{n}^{\prime}\right)=\left[\frac{(n-2)(2 n-6)-(n-4)-1}{2}\right] \leq n^{2}-\frac{11}{2} n+\frac{15}{2}<n^{2}-5 n+4 .
$$

By [10, Theorem 4.1],

$$
\operatorname{ex}\left(2 n-5 ; T_{n}^{*}\right)=\frac{(n-2)(2 n-5)-3(n-4)}{2}=n^{2}-6 n+11<n^{2}-5 n+4 .
$$

Thus, applying Lemma 4.3 we obtain $r\left(T_{n}^{i}, T_{n}\right) \leq 2 n-5$. Hence $r\left(T_{n}^{i}, T_{n}\right)=2 n-5$ as asserted.

Remark 4.1 Let $n \in \mathbb{N}, n \geq 5$ and $i \in\{1,2\}$. From [5, Theorem 3.1(ii)] we know that $r\left(K_{1, n-1}, T_{n}^{i}\right)=2 n-3$.

Theorem 4.3. Let $n \in \mathbb{N}$ and $i \in\{1,2\}$. Then $r\left(P_{n}, T_{n}^{i}\right)=2 n-7$ for $n \geq 17$, $r\left(P_{n-1}, T_{n}^{i}\right)=2 n-7$ for $n \geq 13, r\left(P_{n-2}, T_{n}^{i}\right)=2 n-7$ for $n \geq 11$ and $r\left(P_{n-3}, T_{n}^{i}\right)=2 n-7$ for $n \geq 8$.

Proof. Suppose $n \geq 8$ and $s \in\{0,1,2,3\}$. From Lemma 4.2(ii) we have $r\left(P_{n-s}, T_{n}^{i}\right) \geq$ $2(n-3)-1=2 n-7$. By $(1.1)$,

$$
\operatorname{ex}\left(2 n-7 ; P_{n-s}\right)= \begin{cases}\frac{(n-2)(2 n-7)-5(n-6)}{2}=\frac{(n-4)(2 n-7)+16-n}{2} & \text { if } s=0, \\ \frac{(n-3)(2 n-7)-3(n-5)}{2}=\frac{(n-4)(2 n-7)+8-n}{2} & \text { if } s=1, \\ \frac{(n-4)(2 n-7)-(n-4)}{2} & \text { if } s=2, \\ \frac{(n-5)(2 n-7)-(n-5)}{2}=\frac{(n-4)(2 n-7)+12-3 n}{2} & \text { if } s=3 .\end{cases}
$$

By Theorems 2.1 and 3.1,

$$
\operatorname{ex}\left(2 n-7 ; T_{n}^{i}\right)= \begin{cases}{\left[\frac{(n-4)(2 n-7)}{2}\right]} & \text { if } n \geq 16, \\ \frac{(n-2)(2 n-7)-5(n-6)}{2}=\frac{(n-4)(2 n-7)+16-n}{2} & \text { if } n<16 .\end{cases}
$$

For $n \geq 17,13,11$ or 8 according as $s=0,1,2$ or 3 , from the above we find $\operatorname{ex}(2 n-$ $\left.7 ; P_{n-s}\right)+\operatorname{ex}\left(2 n-7 ; T_{n}^{i}\right)<\left(\begin{array}{c}2 n-7 \\ 2\end{array}\right)$ and so $r\left(P_{n-s}, T_{n}^{i}\right) \leq 2 n-7$ by Lemma 4.1. This completes the proof. 


\section{The Ramsey number $r\left(T_{m}^{i}, T_{n}\right)$ for $m<n$}

Proposition 5.1 (Burr[1]). Let $m, n \in \mathbb{N}$ with $m \geq 3$ and $m-1 \mid n-2$. Let $T_{m}$ be a tree on $m$ vertices. Then $r\left(T_{m}, K_{1, n-1}\right)=m+n-2$.

Proposition 5.2 (Guo and Volkmann [5, Theorem 3.1]). Let $m, n \in \mathbb{N}, m \geq 3$ and $n=k(m-1)+b$ with $k \in \mathbb{N}$ and $b \in\{0,1, \ldots, m-2\} \backslash\{2\}$. Let $T_{m} \neq K_{1, m-1}$ be a tree on $m$ vertices. Then $r\left(T_{m}, K_{1, n-1}\right) \leq m+n-3$. Moreover, if $k \geq m-b$, then $r\left(T_{m}, K_{1, n-1}\right)=m+n-3$.

Lemma 5.1 ([6, Theorem 8.3, pp.11-12]). Let $a, b, n \in \mathbb{N}$. If $a$ is coprime to $b$ and $n \geq(a-1)(b-1)$, then there are two nonnegative integers $x$ and $y$ such that $n=a x+b y$.

Theorem 5.1. Let $m, n \in \mathbb{N}, n>m \geq 5, m-1 \nmid n-2$ and $i \in\{1,2\}$. Then $r\left(T_{m}^{i}, K_{1, n-1}\right)=m+n-3$ or $m+n-4$. Moreover, if $n \geq(m-3)^{2}+1$ or $m+n-4=$ $(m-1) x+(m-2) y$ for some nonnegative integers $x$ and $y$, then $r\left(T_{m}, K_{1, n-1}\right)=m+n-3$ for any tree $T_{m} \neq K_{1, m-1}$ of order $m$.

Proof. Let $T_{m} \neq K_{1, m-1}$ be a tree on $m$ vertices. From Proposition 5.2 we know that $r\left(T_{m}, K_{1, n-1}\right) \leq m+n-3$. By Lemma 4.2(iii), $r\left(T_{m}^{i}, K_{1, n-1}\right) \geq m-3+n-1$. Thus, $r\left(T_{m}^{i}, K_{1, n-1}\right)=m+n-3$ or $m+n-4$. If $n \geq(m-3)^{2}+1$, then $m+n-4 \geq(m-2)(m-3)$ and so $m+n-4=(m-1) x+(m-2) y$ for some nonnegative integers $x$ and $y$ by Lemma 5.1. If $m+n-4=(m-1) x+(m-2) y$ for $x, y \in\{0,1,2, \ldots\}$, setting $G=x K_{m-1} \cup y K_{m-2}$ we see that $G$ does not contain any copies of $T_{m}$ and $\bar{G}$ does not contain any copies of $K_{1, n-1}$. Thus $r\left(T_{m}, K_{1, n-1}\right) \geq 1+|V(G)|=m+n-3$. Now putting all the above together we obtain the theorem.

Theorem 5.2. Let $m, n \in \mathbb{N}, n>m \geq 6, m-1 \mid n-3$ and $i \in\{1,2\}$. Then $r\left(T_{m}^{i}, T_{n}^{\prime}\right)=m+n-3$.

Proof. By Theorems 2.1 and 3.1, ex $\left(m+n-3 ; T_{m}^{i}\right)=\frac{(m-2)(m+n-3)-(m-2)}{2}<\frac{(m-2)(m+n-3)}{2}$. Thus applying $[9$, Theorem 5.1] we obtain the conclusion.

Theorem 5.3. Suppose $i \in\{1,2\}, m, n \in \mathbb{N}, n>m \geq 7$ and $m-1 \nmid(n-3)$. Then $m+n-5 \leq r\left(T_{m}^{i}, T_{n}^{\prime}\right) \leq m+n-4$ and $m+n-6 \leq r\left(T_{m}^{i}, T_{n}^{*}\right) \leq m+n-4$. Moreover, if $n=k(m-1)+b=q(m-2)+a, k, q \in \mathbb{N}, a \in\{0,1, \ldots, m-3\}, b \in\{0,1, \ldots, m-2\}$ and one of the following conditions holds:

(1) $b \in\{1,2,4\}$,

(2) $b=0$ and $k \geq 3$,

(3) $n \geq(m-3)^{2}+2$,

(4) $n \geq m^{2}-1-b(m-2)$,

(5) $\quad a \geq 3$ and $n \geq(a-4)(m-1)+4$,

then $r\left(T_{m}^{i}, T_{n}^{*}\right)=r\left(T_{m}^{i}, T_{n}^{\prime}\right)=m+n-4$.

Proof. By Lemma 4.2 we have $r\left(T_{m}^{i}, T_{n}^{\prime}\right) \geq m-3+n-2$ and $r\left(T_{m}^{i}, T_{n}^{*}\right) \geq m-3+n-3$. Since $m-1 \nmid n-3$, we have $m-1 \nmid m+n-4$. From Corollaries 2.1 and 3.1 we find $\operatorname{ex}\left(m+n-4 ; T_{m}^{i}\right) \leq \frac{(m-2)(m+n-5)}{2}$. Hence, by [9, Lemma 5.2] we have $r\left(T_{m}^{i}, T_{n}^{\prime}\right) \leq m+n-4$, and by $\left[9\right.$, Lemma 4.2] we have $r\left(T_{m}^{i}, T_{n}^{*}\right) \leq m+n-4$. Now applying [9, Theorems 4.4 and 5.4] we deduce the remaining assertion. 


\section{The Ramsey number $r\left(G_{m}, T_{n}^{j}\right)$ for $m<n$}

Theorem 6.1. Let $m, n \in \mathbb{N}, m \geq 5, n \geq 8, n>m$ and $j \in\{1,2\}$. Then $r\left(K_{1, m-1}, T_{n}^{j}\right)$ $=m+n-4$ or $m+n-5$. Moreover, if $2 \mid m n$, then $r\left(K_{1, m-1}, T_{n}^{j}\right)=m+n-4$.

Proof. From Lemma 4.2 we deduce that $r\left(K_{1, m-1}, T_{n}^{j}\right) \geq m-1+n-3-(1-$ $\left.(-1)^{(m-2)(n-4)}\right) / 2=m+n-4-\left(1-(-1)^{m n}\right) / 2$. So, it suffices to prove that $r\left(K_{1, m-1}, T_{n}^{j}\right) \leq$ $m+n-4$. By Lemma 2.1, $\operatorname{ex}\left(m+n-4 ; K_{1, m-1}\right)=\left[\frac{(m-2)(m+n-4)}{2}\right]$. By Theorems 2.1 and 3.1 , we have

$$
\operatorname{ex}\left(m+n-4 ; T_{n}^{j}\right)=\left[\frac{(n-4)(m+n-4)}{2}\right] \text { or } \frac{(n-2)(m+n-4)-(m-3)(n-m+2)}{2} .
$$

Since $\left[\frac{(m-2)(m+n-4)}{2}\right]+\left[\frac{(n-4)(m+n-4)}{2}\right] \leq \frac{(m+n-6)(m+n-4)}{2}<\left(\begin{array}{c}m+n-4 \\ 2\end{array}\right)$ and

$$
\begin{aligned}
& \frac{(m-2)(m+n-4)}{2}+\frac{(n-2)(m+n-4)-(m-3)(n-m+2)}{2} \\
& =\frac{(m+n-4)(m+n-5)-(m-4)\left(n-m-\frac{2}{m-4}\right)}{2}<\left(\begin{array}{c}
m+n-4 \\
2
\end{array}\right),
\end{aligned}
$$

we see that $\operatorname{ex}\left(m+n-4 ; K_{1, m-1}\right)+\operatorname{ex}\left(m+n-4 ; T_{n}^{j}\right)<\left(\begin{array}{c}m+n-4 \\ 2\end{array}\right)$ and so $r\left(K_{1, m-1}, T_{n}^{j}\right) \leq$ $m+n-4$ by Lemma 4.1. This completes the proof.

Theorem 6.2. Let $m, n \in \mathbb{N}, m \geq 4, n \geq 7, m-1 \mid n-4$ and $j \in\{1,2\}$.

(i) If $G_{m}$ is a connected graph of order $m$ with ex $\left(m+n-4 ; G_{m}\right) \leq \frac{(m-2)(m+n-5)}{2}$, then $r\left(G_{m}, T_{n}^{j}\right)=m+n-4$.

(ii) $r\left(T_{m}^{\prime}, T_{n}^{j}\right)=r\left(T_{m}^{1}, T_{n}^{j}\right)=r\left(T_{m}^{2}, T_{n}^{j}\right)=m+n-4$ for $m \geq 5, r\left(T_{m}^{*}, T_{n}^{j}\right)=m+n-4$ for $m \geq 6$, and $r\left(P_{m}, T_{n}^{j}\right)=m+n-4$.

Proof. Set $t=(n-4) /(m-1)$. Suppose that $G_{m}$ is a connected graph of order $m$ with $\operatorname{ex}\left(m+n-4 ; G_{m}\right) \leq \frac{(m-2)(m+n-5)}{2}$. Then clearly $\Delta\left(\overline{(t+1) K_{m-1}}\right)=t(m-1)=n-4$. Thus, $(t+1) K_{m-1}$ does not contain any copies of $G_{m}$ and $\overline{(t+1) K_{m-1}}$ does not contain any copies of $T_{n}^{j}$. Hence $r\left(G_{m}, T_{n}^{j}\right) \geq 1+(t+1)(m-1)=m+n-4$. By Theorems 2.1 and 3.1,

$$
\operatorname{ex}\left(m+n-4 ; T_{n}^{j}\right)=\left[\frac{(n-4)(m+n-4)}{2}\right] \text { or } \frac{(n-2)(m+n-4)-(m-3)(n-m+2)}{2} .
$$

If $\operatorname{ex}\left(m+n-4 ; T_{n}^{j}\right)=\left[\frac{(n-4)(m+n-4)}{2}\right]$, then

$$
\begin{aligned}
& \operatorname{ex}\left(m+n-4 ; G_{m}\right)+\operatorname{ex}\left(m+n-4 ; T_{n}^{j}\right) \\
& \leq \frac{(m-2)(m+n-5)+(n-4)(m+n-4)}{2}<\left(\begin{array}{c}
m+n-4 \\
2
\end{array}\right) .
\end{aligned}
$$

If $\operatorname{ex}\left(m+n-4 ; T_{n}^{j}\right)=\frac{(n-2)(m+n-4)-(m-3)(n-m+2)}{2}$, then

$$
\begin{aligned}
& \operatorname{ex}\left(m+n-4 ; G_{m}\right)+\operatorname{ex}\left(m+n-4 ; T_{n}^{j}\right) \\
& \leq \frac{(m-2)(m+n-5)+(n-2)(m+n-4)-(m-3)(n-m+2)}{2} \\
& =\left(\begin{array}{c}
m+n-4 \\
2
\end{array}\right)-\frac{(m-4)(n-m+1)}{2}<\left(\begin{array}{c}
m+n-4 \\
2
\end{array}\right) .
\end{aligned}
$$


Therefore, by Lemma 4.1 we always have $r\left(G_{m}, T_{n}^{j}\right) \leq m+n-4$ and hence $r\left(G_{m}, T_{n}^{j}\right)=$ $m+n-4$. This proves (i).

Now consider (ii). Note that $m+n-4 \equiv 1(\bmod m-1)$. By $(1.1)$, we have $\operatorname{ex}(m+n-$ $\left.4 ; P_{m}\right)=\frac{(m-2)(m+n-5)}{2}$. By Lemma 4.4, $\operatorname{ex}\left(m+n-4 ; T_{m}^{\prime}\right)=\frac{(m-2)(m+n-5)}{2}$ for $m \geq 5$. By [10, Theorem 4.2], ex $\left(m+n-4 ; T_{m}^{*}\right)=\frac{(m-2)(m+n-5)}{2}$ for $m \geq 6$. By Theorems 2.1 and 3.1, $\operatorname{ex}\left(m+n-4 ; T_{m}^{i}\right)=\frac{(m-2)(m+n-5)}{2}$ for $i \in\{1,2\}$ and $m \geq 5$. Thus from (i) and the above we deduce (ii). The proof is complete.

Lemma 6.1. Let $j \in\{1,2\}, m, n \in \mathbb{N}, m \geq 7$ and $m-1 \nmid n-4$. Assume $n=m+1 \geq 12$ or $n \geq \max \{m+2,19-m\}$.

(i) If $G_{m}$ is a connected graph of order $m$ with ex $\left(m+n-5 ; G_{m}\right) \leq \frac{(m-2)(m+n-6)}{2}$, then $r\left(G_{m}, T_{n}^{j}\right) \leq m+n-5$.

(ii) For $T_{m} \in\left\{P_{m}, T_{m}^{\prime}, T_{m}^{*}, T_{m}^{1}, T_{m}^{2}\right\}$ we have $r\left(T_{m}, T_{n}^{j}\right) \leq m+n-5$.

Proof. Since $m+n-5=n-1+m-4$, by Theorems 2.1 and 3.1 we have

$$
\begin{aligned}
\operatorname{ex}\left(m+n-5 ; T_{n}^{j}\right)= & {\left[\frac{(n-4)(m+n-5)}{2}\right] } \\
& \text { or } \quad \frac{(n-2)(m+n-5)-(m-4)(n-1-(m-4))}{2} .
\end{aligned}
$$

If $n=m+1$, then $(m-4)(n-3-(m-4))=2(n-5)$. If $n \geq m+2$, then $3 \leq m-4 \leq n-6$ and so $(m-4)(n-3-(m-4))=\left(\frac{n-3}{2}\right)^{2}-\left(m-4-\frac{n-3}{2}\right)^{2} \geq\left(\frac{n-3}{2}\right)^{2}-\left(n-6-\frac{n-3}{2}\right)^{2}=3(n-6)$. Thus,

$$
\begin{aligned}
& \frac{(n-4)(m+n-5)+m-2}{2}-\frac{(n-2)(m+n-5)-(m-4)(n-1-(m-4))}{2} \\
& =\frac{(m-4)(n-3-(m-4))-2 n+m}{2} \\
& \geq \begin{cases}\frac{2(n-5)-2 n+m}{2}=\frac{m-10}{2}>0 & \text { if } n=m+1 \geq 12, \\
\frac{3(n-6)-2 n+m}{2}=\frac{n-10+m-8}{2}>0 & \text { if } n \geq \max \{m+2,19-m\} .\end{cases}
\end{aligned}
$$

Therefore, from the above we deduce that

$$
\operatorname{ex}\left(m+n-5 ; T_{n}^{j}\right)<\frac{(n-4)(m+n-5)+m-2}{2} .
$$

Hence, if $G_{m}$ is a connected graph of order $m$ with $\operatorname{ex}\left(m+n-5 ; G_{m}\right) \leq \frac{(m-2)(m+n-6)}{2}$, then

$$
\begin{aligned}
& \operatorname{ex}\left(m+n-5 ; G_{m}\right)+\operatorname{ex}\left(m+n-5 ; T_{n}^{j}\right) \\
& <\frac{(m-2)(m+n-6)}{2}+\frac{(n-4)(m+n-5)+m-2}{2}=\left(\begin{array}{c}
m+n-5 \\
2
\end{array}\right) .
\end{aligned}
$$

Applying Lemma 4.1 we obtain (i).

Now we consider (ii). Since $m-1 \nmid(m+n-5)$, by Corollaries 2.1 and 3.1 we have $\operatorname{ex}\left(m+n-5 ; T_{m}^{i}\right) \leq \frac{(m-2)(m+n-6)}{2}$ for $i \in\{1,2\}$. By $(1.1), \operatorname{ex}\left(m+n-5 ; P_{m}\right) \leq \frac{(m-2)(m+n-6)}{2}$. By Lemma 4.4, $\operatorname{ex}\left(m+n-5 ; T_{m}^{\prime}\right) \leq \frac{(m-2)(m+n-6)}{2}$. By [10, Theorems 4.1-4.5], ex $(m+n-$ $\left.5 ; T_{m}^{*}\right) \leq \frac{(m-2)(m+n-6)}{2}$. Thus, from the above and (i) we deduce (ii). This proves the lemma.

Theorem 6.3. Let $m \in \mathbb{N}$ and $j \in\{1,2\}$. 
(i) We have

$$
r\left(T_{m}^{\prime}, T_{m+1}^{j}\right)= \begin{cases}2 m-4 & \text { if } 2 \nmid m \text { and } m \geq 9, \\ 2 m-5 & \text { if } 2 \mid m \text { and } m \geq 16 .\end{cases}
$$

(ii) If $n \in \mathbb{N}, m \geq 7, n \geq \max \{m+2,19-m\}$ and $m-1 \nmid n-4$, then $r\left(T_{m}^{\prime}, T_{n}^{j}\right)=m+n-5$.

Proof. We first assume $2 \nmid m$ and $m \geq 9$. By Lemma 4.2(i), we have $r\left(T_{m}^{\prime}, T_{m+1}^{j}\right) \geq$ $m-2+m-2=2 m-4$. By Lemma 4.4, $\operatorname{ex}\left(2 m-4 ; T_{m}^{\prime}\right)=\frac{(m-2)(2 m-4)-2(m-3)}{2}=m^{2}-5 m+7$. By Theorems 2.1 and 3.1, $\operatorname{ex}\left(2 m-4 ; T_{m+1}^{j}\right)=\frac{(m-1)(2 m-4)-4(m-4)}{2}=m^{2}-5 m+10$. Thus,

$$
\begin{aligned}
& \operatorname{ex}\left(2 m-4 ; T_{m}^{\prime}\right)+\operatorname{ex}\left(2 m-4 ; T_{m+1}^{j}\right) \\
& =m^{2}-5 m+7+m^{2}-5 m+10=2 m^{2}-10 m+17<2 m^{2}-9 m+10=\left(\begin{array}{c}
2 m-4 \\
2
\end{array}\right) .
\end{aligned}
$$

Hence, by Lemma 4.1 we obtain $r\left(T_{m}^{\prime}, T_{m+1}^{j}\right) \leq 2 m-4$ and so $r\left(T_{m}^{\prime}, T_{m+1}^{j}\right)=2 m-4$.

Now we assume $2 \mid m$ and $m \geq 16$. By Lemma 4.2(i), $r\left(T_{m}^{\prime}, T_{m+1}^{j}\right) \geq m-2+m-2-1=$ $2 m-5$. By Lemma 4.4, ex $\left(2 m-5 ; T_{m}^{\prime}\right)=\left[\frac{(m-2)(2 m-6)-(m-3)}{2}\right]=\frac{2 m^{2}-11 m+14}{2}$. By Theorems 2.1 and 3.1, $\operatorname{ex}\left(2 m-5 ; T_{m+1}^{j}\right)=\left[\frac{(m-1)(2 m-5)}{2}\right]-(2 m-5)=\frac{2 m^{2}-11 m+14}{2}$. Thus,

$$
\operatorname{ex}\left(2 m-5 ; T_{m}^{\prime}\right)+\operatorname{ex}\left(2 m-5 ; T_{m+1}^{j}\right)=2 m^{2}-11 m+14<2 m^{2}-11 m+15=\left(\begin{array}{c}
2 m-5 \\
2
\end{array}\right) .
$$

Hence, by Lemma 4.1 we obtain $r\left(T_{m}^{\prime}, T_{m+1}^{j}\right) \leq 2 m-5$ and so $r\left(T_{m}^{\prime}, T_{m+1}^{j}\right)=2 m-5$. This proves (i).

Now we consider (ii). Suppose $n \in \mathbb{N}, m \geq 7$ and $n \geq \max \{m+2,19-m\}$. By Lemma 6.1(ii), $r\left(T_{m}^{\prime}, T_{n}^{j}\right) \leq m+n-5$. By Lemma 4.2, we have $r\left(T_{m}^{\prime}, T_{n}^{j}\right) \geq m-2+n-3$. Thus, $r\left(T_{m}^{\prime}, T_{n}^{j}\right)=m+n-5$. This proves (ii). The proof is complete.

Theorem 6.4. Let $j \in\{1,2\}, m, n \in \mathbb{N}, m \geq 7$ and $m-1 \nmid n-4$. Suppose $n=m+1 \geq 12$ or $n \geq \max \{m+2,19-m\}$. Assume that $G_{m} \in\left\{P_{m}, T_{m}^{*}, T_{m}^{1}, T_{m}^{2}\right\}$ or $G_{m}$ is a connected graph of order $m$ such that $\operatorname{ex}\left(m+n-5 ; G_{m}\right) \leq \frac{(m-2)(m+n-6)}{2}$. If $n \geq(m-3)^{2}+3$ or $m+n-6=(m-1) x+(m-2) y$ for some nonnegative integers $x$ and $y$, then $r\left(G_{m}, T_{n}^{j}\right)=$ $m+n-5$.

Proof. If $n \geq(m-3)^{2}+3$, then $m+n-6 \geq(m-2)(m-3)$ and so $m+n-6=$ $(m-1) x+(m-2) y$ for some $x, y \in\{0,1,2, \ldots\}$ by Lemma 5.1. Now suppose $m+n-6=$ $(m-1) x+(m-2) y$, where $x, y \in\{0,1,2, \ldots\}$. Set $G=x K_{m-1} \cup y K_{m-2}$. Then $\Delta(\bar{G}) \leq n-4$. Thus, $G$ does not contain any copies of $G_{m}$ and $\bar{G}$ does not contain any copies of $T_{n}^{j}$. Hence $r\left(G_{m}, T_{n}^{j}\right) \geq 1+|V(G)|=m+n-5$. On the other hand, by Lemma 6.1 we have $r\left(G_{m}, T_{n}^{j}\right) \leq m+n-5$. Thus $r\left(G_{m}, T_{n}^{j}\right)=m+n-5$. This proves the theorem.

Corollary 6.1. Let $m, n \in \mathbb{N}, m \geq 7, m-1 \mid n-b, b \in\{2,3,5\}, n \geq \max \{m+2,19-m\}$ and $j \in\{1,2\}$. Assume that $G_{m} \in\left\{P_{m}, T_{m}^{*}, T_{m}^{1}, T_{m}^{2}\right\}$ or $G_{m}$ is a connected graph of order $m$ with ex $\left(m+n-5 ; G_{m}\right) \leq \frac{(m-2)(m+n-6)}{2}$. Then $r\left(G_{m}, T_{n}^{j}\right)=m+n-5$.

Proof. Set $k=(n-b) /(m-1)$. Then $k \in \mathbb{N}$. For $b=2$ we have $k \geq 2$. Since

$$
m+n-6= \begin{cases}(k-2)(m-1)+3(m-2) & \text { if } b=2, \\ (k-1)(m-1)+2(m-2) & \text { if } b=3, \\ (k+1)(m-1) & \text { if } b=5,\end{cases}
$$

the result follows from Theorem 6.4. 
Theorem 6.5. Let $m \in \mathbb{N}, m \geq 12$ and $i, j \in\{1,2\}$. Then

$$
r\left(T_{m}^{i}, T_{m+1}^{j}\right)=r\left(T_{m}^{*}, T_{m+1}^{j}\right)=2 m-5 .
$$

Proof. Let $T_{m} \in\left\{T_{m}^{i}, T_{m}^{*}\right\}$. By Theorems 2.1, 3.1 and [10, Theorem 4.1],

$$
\begin{aligned}
& \operatorname{ex}\left(2 m-5 ; T_{m}\right)=\frac{(m-2)(2 m-5)-3(m-4)}{2}, \\
& \operatorname{ex}\left(2 m-5 ; T_{m+1}^{j}\right)=\frac{(m-1)(2 m-5)-5(m-5)}{2} \text { or }\left[\frac{(m-3)(2 m-5)}{2}\right] .
\end{aligned}
$$

Since $\frac{(m-2)(2 m-5)-3(m-4)}{2}+\frac{(m-3)(2 m-5)}{2}=\frac{(2 m-5)(2 m-6)+7-m}{2}<\left(\begin{array}{c}2 m-5 \\ 2\end{array}\right)$ and

$$
\begin{aligned}
& \frac{(m-2)(2 m-5)-3(m-4)}{2}+\frac{(m-1)(2 m-5)-5(m-5)}{2} \\
& =2 m^{2}-12 m+26<2 m^{2}-11 m+15=\left(\begin{array}{c}
2 m-5 \\
2
\end{array}\right),
\end{aligned}
$$

we see that $\operatorname{ex}\left(2 m-5 ; T_{m}\right)+\operatorname{ex}\left(2 m-5 ; T_{m+1}^{j}\right)<\left(\begin{array}{c}2 m-5 \\ 2\end{array}\right)$. Hence, applying Lemma 4.1 we deduce that $r\left(T_{m}, T_{m+1}^{j}\right) \leq 2 m-5$. Since $\Delta\left(T_{m}\right)=m-3$ and $\Delta\left(T_{m+1}^{j}\right)=m-2$, by Lemma 4.2(i) we have $r\left(T_{m}, T_{m+1}^{j}\right) \geq m-3+m-2=2 m-5$. Hence $r\left(T_{m}, T_{m+1}^{j}\right)=2 m-5$. This proves the theorem.

Acknowledgements. The first author is supported by the National Natural Science Foundation of China (grant No. 11371163), and the second author is supported by the Fundamental Research Funds for the Central Universities (grant No. 2014QNA58).

\section{References}

[1] S.A. Burr, Generalized Ramsey theory for graphs-a survey, in: Graphs and Combinatorics, R.A. Bari and F. Harary (eds.), Lecture Notes in Math. 406, Springer, Berlin, $1974,52-75$.

[2] S.A. Burr and P. Erdős, Extremal Ramsey theory for graphs, Util. Math. 9(1976), 247-258.

[3] S.A. Burr and J.A. Roberts, On Ramsey numbers for stars, Util. Math. 4(1973), 217220 .

[4] R.J. Faudree and R.H. Schelp, Path Ramsey numbers in multicolorings, J. Combin. Theory Ser. B 19(1975), 150-160.

[5] Y. Guo and L. Volkmann, Tree-Ramsey numbers, Australas. J. Combin. 11(1995), 169-175.

[6] L.K. Hua, Introduction to Number Theory, Springer, Berlin, 1982 (translated from the Chinese by P. Shiu).

[7] S.P. Radziszowski, Small Ramsey numbers, Revision \#14, Electron. J. Combin. 2014, Dynamic Survey DS1, 94pp. 
[8] A.F. Sidorenko, Asymptotic solution for a new class of forbidden r-graphs, Combinatorica 9(1989), 207-215.

[9] Z.H. Sun, Ramsey numbers for trees, Bull. Austral. Math. Soc. 86(2012), 164-176.

[10] Z.H. Sun and L.L.Wang, Turán's problem for trees, J. Combin. Number Theory 3(2011), 51-69.

[11] M. Woźniak, On the Erdős-Sós conjecture, J. Graph Theory 21(1996), 229-234. 